\title{
Direct Measurement of Glycosaminoglycan Intermolecular Interactions via High-Resolution Force Spectroscopy
}

\author{
J . Seog, ${ }^{\dagger}$ D. Dean, ${ }^{\ddagger}$ A. H. K. Plaas, ${ }^{\prime}$ S. Wong-Palms," A. J . Grodzinsky, ${ }^{\dagger \neq}$ and \\ C. Ortiz $z^{*, \S}$
}

Departments of Mechanical Engineering, Electrical Engineering and Computer Science, and Materials Science and Engineering, Massachusetts Institute of Technology, 77 Massachusetts Avenue, Cambridge, Massachusetts 02139; and Department of Biochemistry, University of South Florida, 4202 E. Fowler Avenue, Tampa, Florida 33620

Received December 11, 2001; Revised Manuscript Received April 16, 2002

\begin{abstract}
Intermolecular repulsion forces between negatively charged glycosaminoglycan (CS-GAG) macromolecules are a major determinant of cartilage biomechanical properties. It is thought that the el ectrostatic component of the total intermolecular interaction is responsible for $50-75 \%$ of the equilibrium elastic modulus of cartilage in compression, while other forces (e.g., steric, hydration, van der Waals, etc.) may also play a role. To investigate these forces, radiol abeled CS-GAG polymer chains, with a fully extended contour length of $35 \mathrm{~nm}$, were chemically end-grafted to a planar surface to form model biomimetic polyelectrolyte "brush" layers whose environment (e.g., ionic strength, pH) was varied to mimic physiological conditions. The total intersurface force $(\leq n N)$ between the CS-GAG brushes and chemically modified probe tips $\left(\mathrm{SO}_{3}{ }^{-}\right.$and $\mathrm{OH}$ ) was measured as a function of tip-substrate separation distance in aqueous solution using the technique of high-resolution force spectroscopy (HRFS). These experiments showed long-range, nonlinear, purely repulsive forces that decreased in magnitude and range with increasing ionic strength and decreasing $\mathrm{pH}$. To estimate the contribution of the electrostatic component to the total intersurface force, the data were compared to a theoretical model of electrical double layer repulsion based on the Poisson-Boltzmann formulation. The CS-GAG brush layer was approximated as either a flat surface charge density or a smoothed volume of known fixed charge density and the probe tip was modeled as a smooth hemisphere of constant surface charge density. Modeling the CSGAG brush as a volume charge yielded theoretical fits much closer to the experimental data and is a good first step toward deconvolution of the force components.
\end{abstract}

\section{Introduction}

Cartilage is a highly specialized, dense connective tissue found between the surfaces of movable articular joints whose main function is to bear stresses during joint motion. This complex biocomposite possesses high stiffness, toughness, strength, resiliency, and shock absorption. The extracellular matrix (ECM) of cartilage is composed of many different mol ecules and structures. However, the chondroitin sulfate glycosaminoglycan (CS-GAG) chains of the proteoglycan, aggrecan ( $F$ igure la,b), within the ECM, are the major determinant of the tissue's ability to resist compressive loading in vivo (e.g., responsible for $>50 \%$ of the equilibrium compressive el astic modulus under normal physiological conditions ( $0.15 \mathrm{M}$ salt concentration). ${ }^{1} \mathrm{~A}$ single aggrecan molecule contains about $100 \mathrm{CS}-$ GAGs that are covalently bound, at extremely high densities ( $\sim 2-4 \mathrm{~nm}$ molecular separation distance), to a $250 \mathrm{kDa}$ protein core (F igure 1b). Multiple aggrecan molecules assemble further to form supramolecular proteoglycan aggregates by noncovalently attaching to hyaluronic acid polymers, an interaction stabilized by the adjacent binding of link protein (Figure 1a). The aggregates form the gellike

\footnotetext{
* Corresponding author: Department of Materials Science and Engineering, Massachusetts I nstitute of Technology, RM 13-4022, 77 Massachusetts Avenue, Cambridge MA 02139.

† Department of Mechanical Engineering, Massachusetts Institute of Technology.

‡ Department of Electrical Engineering and Computer Science, Massachusetts Institute of Technology.

$\S$ Department of Materials Science and Engineering, Massachusetts Institute of Technology.

"University of South Florida.
}

component of cartilage that is enmeshed within a network of reinforcing collagen fibrils.

CS-GAGs (Figure 1c) are negatively charged, linear polyelectrolytes composed of between 10 and 50 repeats of the disaccharide ( $\mathrm{N}$-acetyl-galactosamine and glucuronic acid) which are extensively substituted with sulfate esters at carbons 4 or 6 of the hexosamine residues. ${ }^{1-3}$ As part of the aggrecan macromolecule, individual CS-GAGs have the tendency to assume an extended, rodlike conformation rather than a random coil under normal physiological conditions of $0.15 \mathrm{M}$ salt concentration due to intramol ecular el ectrostatic repulsion between neighboring negatively charged carboxylate and sulfate groups (i.e., intercharge distance $=0.64$ $\mathrm{nm}),{ }^{3}$ as well as the high chain packing density.

The goal of this research is to determine the underlying molecular mechanisms responsible for the macroscopic compressive biomechanical stiffness of cartilage. It is thought that the unique nanomechanical properties of the constituent CS-GAGs are one of the major determinants of cartilage's biomechanical properties: in particular, electrostatic repulsion due to the electrical double layer as well as macromolecular "steric" or "overlap" repulsion (Figure 2a). ${ }^{4}$ To quantify these molecular-level interactions, CS-GAG polymer chains were chemically end-grafted to planar surfaces to form model biomimetic polyelectrolyte "brushes" whose environment was varied to mimic physiological conditions. The nanoscale surface properties of the CS-GAG brushes were then measured directly in aqueous solutions of different ionic strength (IS) and pH using the technique of high-resolution force spectroscopy (HRFS) which employs an extremely soft microfabricated cantilever 
(a) Proteoglycan aggregate

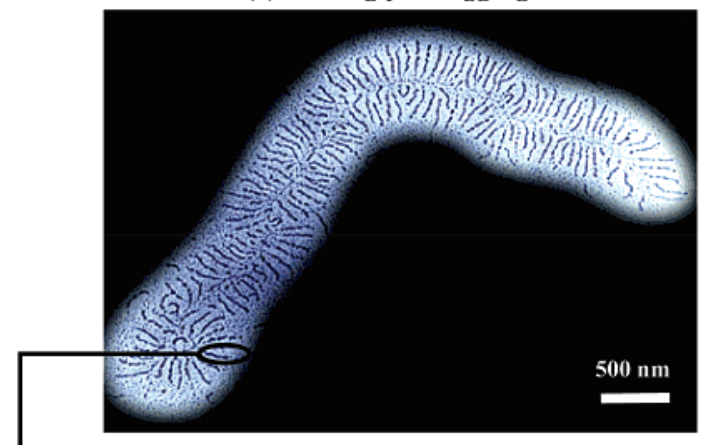

(b) Aggrecan

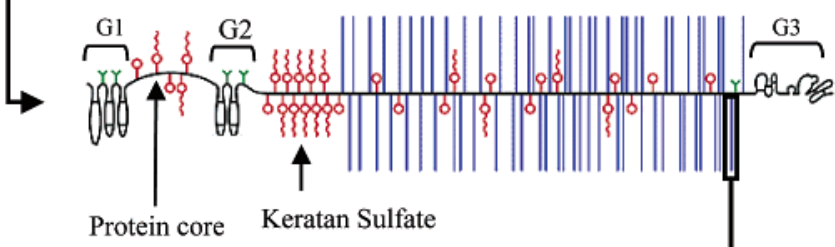

(c) Chondroitin-4-sulfate

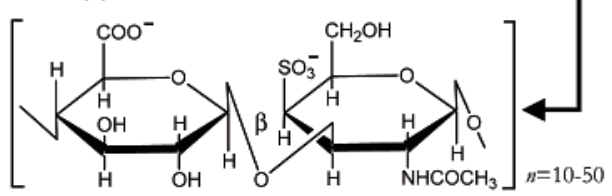

Figure 1. Structural hierarchy of aggrecan in cartilage: (a) electron microscopy of aggrecan and the aggregates it forms with hyaluronan and link protein; ${ }^{79}$ (b) aggrecan core protein containing three globular domains (G1, G2, G3), the CS-GAG attachment region being composed of a variable keratan sulfate region and a chondroitin sulfate region distinguished by their sequence patterns; ${ }^{80}$ (c) chemical structure of the disaccharide repeating unit in chondroitin-4-sulfate glycosaminoglycan (CS-GAG), used in the study.

and probe tip (in our case, chemically modified to known surface chemistry) as a force transducer to record force, $\mathrm{F}(\mathrm{nN})$, vs tip-surface separation distance, D $(\mathrm{nm})$ (Figure 2b). Our long-term objective is to generalize these methods to address a hierarchy of unsolved nanomechanical problems critical to the understanding of molecular structure/function relations in cartilage and other connective tissues. It should also be noted that apart from this broader biological significance, polyelectrolyte brush systems have technological relevance in many other areas of biophysics, polymer physics, surface science, and surfactant science, such as polymerically stabilized colloids, the interaction between membrane proteins and foreign bodies, and surface modification for adhesion and lubrication.

\section{Materials and Methods}

Chemical Modification and Characterization of Substrates and Cantilever/Probe Tips. (a) Substrates. Silicon (100) wafers (Recticon Enterprises, Inc., Pottstown, PA; test grade) were cleaned with ethanol and immediately coated with $2 \mathrm{~nm}$ of chromium to promote adhesion, followed by $100 \mathrm{~nm}$ of Au deposited using a thermal evaporator at $1.5 \AA / \mathrm{s}$ at room temperature at a typical pressure of $2 \times 10^{-6}$ Torr. An atomic force microscope (AFM) topographic image (Digital Instruments Multimode, Santa Barbara, CA) taken in contact mode in air on $1 \mu \mathrm{m}^{2}$ regi on of the Au-coated silicon wafer substrate (Figure 3a) shows a polycrystalline surface structure with a typical root-mean-square (rms) surface roughness of $2 \mathrm{~nm}$ and a gold island size range of 25-76 nm (courtesy of M. Rixman). The Au-coated silicon wafers were cleaned using piranha
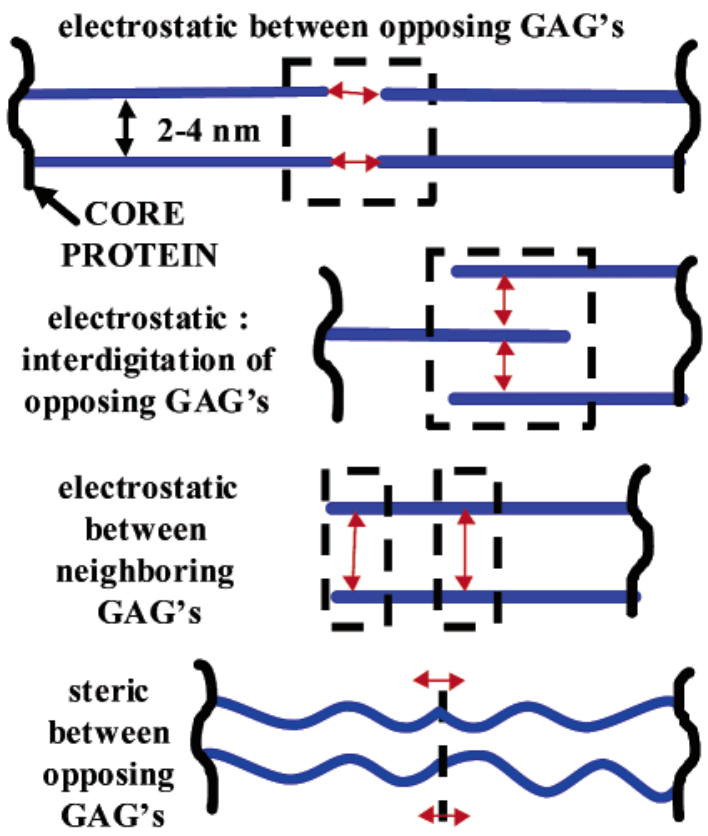

(a)

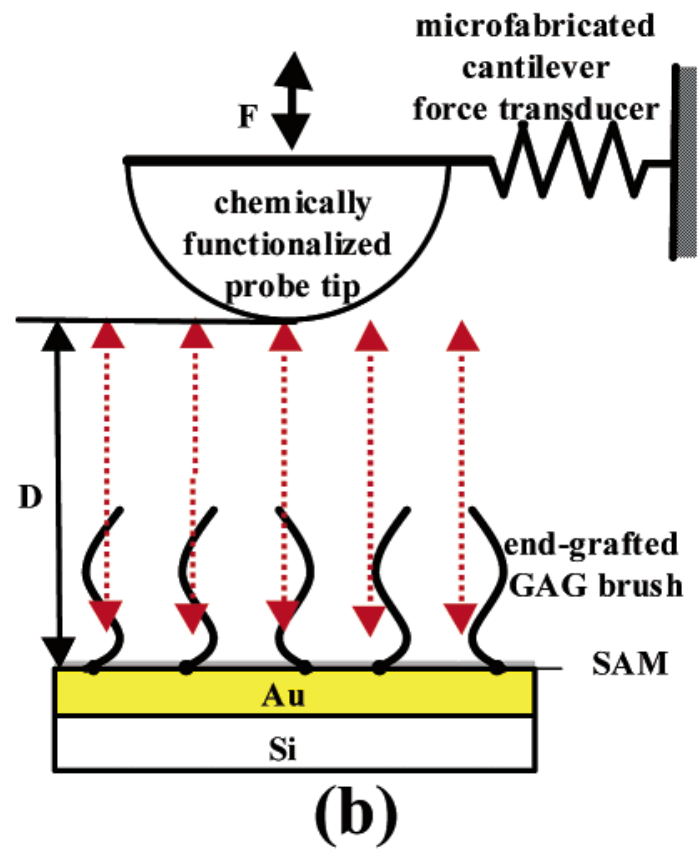

Figure 2. (a) Possible components of total CS-GAG andCSGAG intermolecular interaction. (b) Schematic diagram of high-resolution force spectroscopy experiment of chemically functionalized probe tip vs end-grafted CS-GAG polymer brush.

solution (3:1 concentrated $\mathrm{H}_{2} \mathrm{SO}_{4} / \mathrm{H}_{2} \mathrm{O}_{2}(30 \%)$ ) for 5 min just before further chemical modification. WARNING! Piranha solution is extremely oxidizing, reacts violently with organics, and should be only be stored in loosely tightened containers to avoid buildup of pressure. Sulfate-functionalized surfaces were produced for control experiments by immersion in a $5 \mathrm{mM}$ ethanol solution of 2-mercaptoethanesulfonic acid sodium salt, $\mathrm{HS}\left(\mathrm{CH}_{2}\right)_{2} \mathrm{SO}_{3} \mathrm{Na}$ (Aldrich), for $24 \mathrm{~h}$ and then backfilled with ethanethiol, $\mathrm{HS}\left(\mathrm{CH}_{2}\right) \mathrm{CH}_{3}$ (Aldrich), also by immersion in a 5 $\mathrm{mM}$ ethanol solution for $30 \mathrm{~min}$.

(b) Cantilever/Probe Tips. $\mathrm{Si}_{3} \mathrm{~N}_{4}$ V-shaped cantilever/ probe tips (Thermomicroscopes, Inc., Sunnyvale, CA; unsharpened Microl evers) were al so coated with $2 \mathrm{~nm}$ of chromium to promote adhesion, followed by $50 \mathrm{~nm}$ of Au deposited using a 


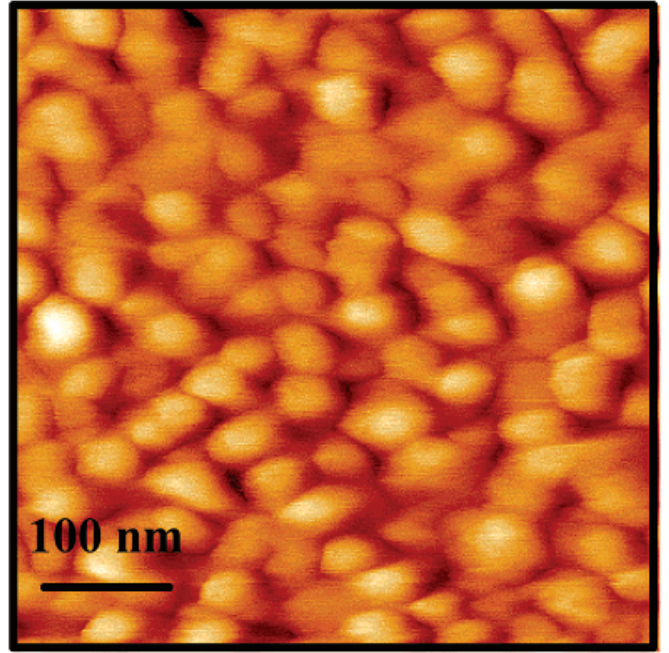

(a)

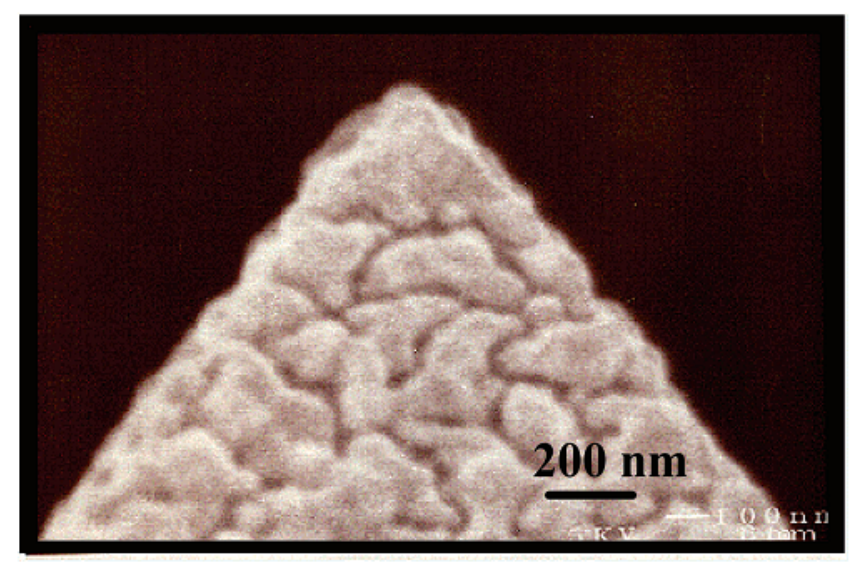

(b)

Figure 3. (a) Contact mode atomic force microscope topographic image of evaporated gold on silicon wafer taken in air. Root-mean-square value for surface roughness $\approx 2.2 \mathrm{~nm}$. (b) Scanning electron micrograph of gold-coated $\mathrm{Si}_{3} \mathrm{~N}_{4}$ probe tip. Probe tip radius $\approx 127 \mathrm{~nm}$.

thermal evaporator at $1.5 \AA / s$ at room temperature. Figure 3b shows a scanning electron microscope (SEM) image (J EOL 6320FV field-emission high-resolution SEM image of the polycrystalline gold-coated probe tip. This image is representative of the probe tips used for the HRFS experiments reported in this paper. The individual gold grain at the tip apex defines the radius of curvature of the tip, $\mathrm{R}_{\mathrm{tip}}$, and was determined by drawing two intersecting straight lines tangential to the edges of the probe tip and then drawing a circle tangential to both of them. The radius of this circle was assumed to be $R_{\text {tip. }}$. The radii of curvature were found to be $\sim 127 \mathrm{~nm}$ (for the sulfatemodified probe tip used on the sulfate-modified surface as a function of IS), $\sim 25 \mathrm{~nm}$ (for the sulfate-modified probe tip used on the CS-GAG-modified surface as a function of IS), 117 $\mathrm{nm}$ (for the sulfate-modified probe tip on the CS-GAGmodified surface as a function of $\mathrm{pH}$ ), and $\sim 25 \mathrm{~nm}$ (for the hydroxy-modified probetip on the CS-GAG-modified surface as a function of IS). The Au-coated probe tips were sulfate- or hydroxy-functionalized by reaction with 2-mercaptoethanesulfonic acid sodium salt (Aldrich) or 11-mercaptoundecanol, $\mathrm{HS}\left(\mathrm{CH}_{2}\right)_{11} \mathrm{OH}$ (donated by Prof. P. Laibinis, MIT, Chemical Engineering), respectively, by immersion of the cantilever probe tips in $5 \mathrm{mM}$ ethanol solutions for $24 \mathrm{~h}$. The probe tips that were modified with 2-mercaptoethanesulfonic acid sodium salt were again backfilled with ethanethiol (Aldrich) by immersion in a $5 \mathrm{mM}$ ethanol solution for $30 \mathrm{~min}$.

Preparation and Characterization of End-Grafted CS-GAG Brush Layer. In the literature, end-grafted polyelectrolyte brushes have been successfully prepared by four different techniques: (1) "grafting to" where (mono)endfunctional ized polymer chains are chemisorbed onto a surface from solution, ${ }^{5}$ (2) "grafting from" where the polymer chains are generated directly at the surface in situ, for example, by using a self-assembled monolayer (SAM) of initiator already covalently attached to the surface to start a radical or anionic polymerization, ${ }^{6,7}$ (3) by using hydrophobically modified polyel ectrolyte block copolymers, ${ }^{8}$ and (4) by a Langmuir-Blodgett (LB) technique using polymer-based amphiphile. ${ }^{9}$ Since the CS-GAG molecules could be prepared in (mono)end-functionalized form (with terminal reactive amine groups), the "grafting to" method was employed in these studies. To create a biomimetic polymer system with configurations similar to that found in aggrecan, we attempted to achieve the highest surface grafting density possible by varying the environmental solution conditions of chemisorption (e.g., ionic strength, pH, CS-GAG concentration, incubation time, etc.). It should be noted that the "grafting to" method to produce high-density brushes is much more challenging experimentally than the "grafting from" or LB methods because initially chemisorbed polymer chains on the surface may shield new incoming chains from accessing the underlying surface below due to a variety of intermolecular repulsive interactions (e.g., steric, el ectrostatic).

Metabolically radiolabeled ${ }^{35} \mathrm{~S}$-aggrecans were prepared from rat chondrosarcoma cell cultures, digested with proteinase $\mathrm{K}$, and the resulting amino acid-terminated ${ }^{35} \mathrm{~S}-\mathrm{CS}-\mathrm{GAG}$ chains were precipitated with ethanol $(75 \%, v / v$ in $0.001 \mathrm{M}$ sodium acetate), purified by Superose 6 (HR10/60) gel filtration chromatography, ${ }^{10}$ and resuspended in $0.01 \mathrm{M}$ phosphate buffer, $\mathrm{pH}=7.4$ at $0.1 \mathrm{mg} / \mathrm{mL}$ concentration. The molecular weight of the ${ }^{35} \mathrm{~S}-\mathrm{CS}-\mathrm{GAG}$ chains was measured to be 13428 by HPLC (Hewlett-Packard 1090, Palo Alto, CA, using a ToyoPearl HW40F column, $50 \mathrm{~cm} \times 8 \mathrm{~mm}$ i.d. $\left.{ }^{11}\right)$, and the polydispersity was calculated from the chromatogram to be 1.06 . The average number of disaccharide units per chain calculated from this molecular weight value was 25 . Since the disaccharide monomer unit length is $1.28 \mathrm{~nm}$, the contour length, $\mathrm{L}_{\text {contour, }}$ was calculated to be $35 \mathrm{~nm}$, which includes a $3 \mathrm{~nm}$ linkage region containing carbohydrate and amino acid moieties. The ${ }^{35} \mathrm{~S}-\mathrm{CS}-\mathrm{GAG}$ chains with their terminal reactive amine groups were treated with $1 \mu \mathrm{M}$ of dithiobis(sulfosuccinimidyl propionate) (DTSSP, Pierce), and the terminal disulfide bond was reduced to a thiol group using $0.1 \mathrm{mM}$ dithiothreitol (DTT, Pierce) (F igure 4). After removal of excess reactants using centrifugal filters (Centricon, Millipore, 3000 MW cutoff), $10 \mu \mathrm{L}$ aliquots of the thiol-terminated ${ }^{35} \mathrm{~S}-\mathrm{CS}-$ GAG chains were placed on piranha-treated $1 \mathrm{~cm} \times 1 \mathrm{~cm}$ goldcoated silicon wafers and incubated in humidity chamber. A reaction time of $72 \mathrm{~h}$ produced the highest grafting density surfaces. After being rinsed, the wafers were placed in $1 \mathrm{mM}$ $\mathrm{C}_{12} \mathrm{H}_{25}-\mathrm{SH}$ solution for $30 \mathrm{~min}$ to passivate that part of the gold surface not modified with CS-GAG. After HRFS experiments were compl eted, the CS-GAG surface grafting density on the substrate was assessed by removal of the ${ }^{35} \mathrm{~S}-\mathrm{CS}-\mathrm{GAG}$ via sonication at $90{ }^{\circ} \mathrm{C}$ and then scintillation counting (i.e., an assay in which the radioactively label ed ${ }^{35}$ S-CS-GAG was mixed with a scintillant and the radioactive decay caused emission of fluorescent light from the scintillant which was then detected and recorded). The CS-GAG surface grafting density was calculated from the total ${ }^{35}$ S-radioactivity of the detached CS-GAG (using the known specific activity of 77317 $\mathrm{cpm} / \mu \mathrm{g}$ of $\left.{ }^{35} \mathrm{~S}-\mathrm{CS}-\mathrm{GAG}\right)$, and was found to be $\Gamma=0.024$ chains/ $\mathrm{nm}^{2}$ or $\sim(6.5 \mathrm{~nm} \times 6.5 \mathrm{~nm})$ area per chain.

High-Resolution Force Spectroscopy (HRFS) Measurements. (a) Instrumentation. HRFS experiments were conducted using a new cantilever-based, piconewton-sensitive instrument; the molecular force probe (MFP) (Asylum Research, Santa Barbara, CA) to measure force, $F(n N)$, vs tipsample separation distance, D (nm). Modeled on AFM technology, the MFP employs a micromachined soft, flexible cantilever with a sharp tip as a force transducer that deflects 

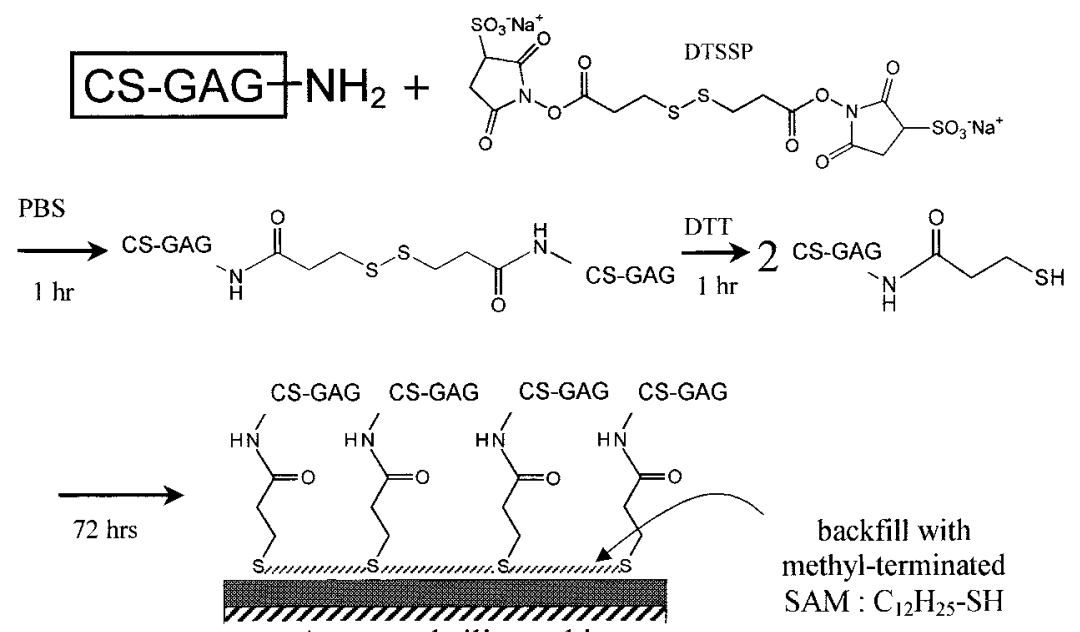

Au-coated silicon chip

Figure 4. Chemical scheme for end-grafting chondroitin-4-sulfate CS-GAG polymer chains to a gold-coated surface.

in response to the small forces between the cantilever tip and a sample surface. A near-IR laser beam is focused on the backside of the end of the cantilever and directed with a mirror into a split position-sensitive photodiode (PSPD). The MFP has an open fluid cell design with an optical (video) microscope located in the base, making it ideal to work on polymeric and biological samples. An adjustable laser focus, novel optic lever geometry, and a low coherence light source optimize response and mi nimize interference reflections from reflective samples. A piezoelectric translator (10 $\mu \mathrm{m}$ range) located on a flexure plate in the head incrementally moves the tip toward the sample in the z-direction perpendicular to sample plane ("approach") and away from the sample ("retract") at a constant rate. A LVDT (linearly variable differential transformer) position sensor ( $<3 \AA$ noise in $0.1-1 \mathrm{kHz}$ bandwidth, $15 \mu \mathrm{m}$ travel, $0.02 \%$ linearity), also located on the flexure plate in the head, quantifies the distance the z-piezo moves the cantilever directly, thus eliminating error due to piezo hysteresis and other nonlinearities and also reducing or eliminating the effects of thermal drift over long time scales.

(b) Conversion of Raw Data and Measurement Errors. Igor Pro software routines (Wavemetrics) were used for conversion of photodiode sensor output voltage (V) into force, $\mathrm{F}(\mathrm{nN})$, and LVDT output (nm) into tip-sample separation distance, D $(\mathrm{nm})$. The vertical sensor outputs (V) (i.e., the difference of the top minus bottom quadrants of the PSPD normalized by the total PSPD output, $s(V)=(T-B) /(T+B))$, were converted into cantilever deflection, $\delta(\mathrm{nm})$. This was implemented assuming that the change in z-piezo position $\mathrm{dz}$ measured by the LVDT (equal to the change in position of the base of the probe tip) is equival ent to the change in cantilever deflection, $\mathrm{d} \delta(\mathrm{nm})$, in the repulsive, contact regime of constant compliance:

$$
\delta(\mathrm{nm})=\mathrm{s}(\mathrm{V}) \times \operatorname{IOLS}(\mathrm{nm} / \mathrm{V})
$$

where the IOLS is the "inverse optical lever sensitivity" (nm/ V) and is equal to the inverse slope of sensor vs LVDT output curve in the constant compliance regime. The force was then calculated by using Hooke's law for a linear elastic spring:

$$
\mathrm{F}(\mathrm{nN})=\mathrm{k}_{\mathrm{c}}(\mathrm{N} / \mathrm{m}) \times \delta(\mathrm{nm})
$$

where $\mathrm{F}(\mathrm{nN})$ is the interaction force and $\mathrm{k}_{\mathrm{c}}$ is the cantilever spring constant $(\mathrm{N} / \mathrm{m}) . \mathrm{k}_{\mathrm{c}}$ was determined for each individual cantilever according to a nondestructive method described in the literature. ${ }^{12}$ The usual convention of positive (+) for repulsive forces and negative (-) for attractive forces was employed. The zero force baseline was taken from data obtained when the cantilever was undeflected far away from the surface $(\sim 1000 \mathrm{~nm})$. The error in force measurements is due to calculation of the IOLS $( \pm 5 \%)$, the spring constant calibration ( $\pm 20 \%$ ), and nonlinearities of the photodetector associated with the finite size of the laser spot $( \pm 2 \%)$. Using the Thermomicroscopes probe tips (V-shaped, nominal tip radius $\sim 20-50 \mathrm{~nm}$, length $=320 \mu \mathrm{m}$, nominal spring constant $\mathrm{k}=0.01 \mathrm{~N} / \mathrm{m}$ ), the MFP is able to achieve a thermally limited force detection of $\sim 5 \mathrm{pN}$ in aqueous solution in a $1 \mathrm{kHz}$ bandwidth. ${ }^{13}$

The LVDT signal output (V) was converted into z-piezo distance, $z(\mathrm{~nm})$, by calibration at Asylum Research, Inc. via interferometry. The LVDT was found to have a sensitivity of $1.47 \mu \mathrm{m} N . z(\mathrm{~nm})$ was converted into the tip-sample separation distance, $D(\mathrm{~nm})$, by correcting for the cantilever displacement due to the surface interaction force:

$$
D(n m)=z(n m)-\delta(n m)
$$

where $\delta$ is calculated from eq 2. The vertical region of apparent infinite slope in the high-force, constant compliance regime was set to $D=0$, which is due to the fact that the spring constant of the cantilever is much less than the stiffness of the substrate. For high-density polymer brushes, it has been shown that there is an inherent error in this assumption due to the presence of a compacted, incompressible polymer layer that is approximately equal to the thickness of the polymer layer in the dry state for lower molecular weights $\left(M_{n} \leq\right.$ 25 000). ${ }^{14,15} \mathrm{~F}$ or the CS-GAG brushes prepared as described above, the thickness in the dry state was measured via ellipsometry and found to be $\sim 3.18 \mathrm{~nm}$. We also used another technique to determine the incompressible layer thickness which involves end-grafting CS-GAG polymer chains to a micrometer-scale chemically patterned surface (prepared via microcontact printing ${ }^{16}$ ) and then using AFM isoforce imaging in the constant compliance region of the force vs distance regime to measure the height of the CS-GAG polymer layer, thus locating the $\mathrm{D}=0$ point. The results yielded an incompressible CS-GAG layer thickness of $\sim 1.5 \mathrm{~nm}$ in air, thinner than the dry layer thickness measured via ellipsometry and the surface chemical patterns were undetectable in all ionic strength buffer solutions, indicating that the incompressible CS-GAG Iayer thickness was negl igible. E ach force vs distance curve was obtained by averaging 10-20 curves from two to three different spots at each condition. The standard deviation of the force was less than $\sim 30 \mathrm{pN}$ for all ionic strength conditions and separation distances measured.

(c) HRFS Experiments. Force vs distance between CSGAG polymer brush surfaces and probe tips was measured in $0.0001 \mathrm{M}$ to $1.0 \mathrm{M} \mathrm{NaCl}$ solutions, $\mathrm{pH} \sim 5.6$, using the MFP at room temperature (with z-piezo velocity $v=0.5-1.0 \mu \mathrm{m} / \mathrm{s}$, z-piezo range $=0.5-1 \mu \mathrm{m}$, and rate of data acquisition $=4000$ points/s). The $\mathrm{NaCl}$ solutions were prepared by mixing $\mathrm{NaCl}$ (Mallinckrodt) and deionized (DI) $\mathrm{H}_{2} \mathrm{O}(18 \mathrm{M} \Omega \cdot \mathrm{cm}$ resistivity, Purelab Plus UV/UF, US Filter, Lowell, MA). Control studies 
were performed between sulfate functionalized tips and sulfate functionalized substrates. Force vs distance using CS-GAG brush substrate was al so measured at different ionic strength and $\mathrm{pH}$ values using tips that were sulfate or hydroxyfunctionalized. In this paper, we focused on data obtained during the approach of the tip to the surface, since we were initially interested in the molecular origins of compressive mechanical properties.

\section{Results}

Control Experiments: Interaction between Sulfate-Functionalized Probe Tip and SulfateFunctionalized Substrate as a Function of Ionic Strength. Figure 5a shows a series of (average) force $(\mathrm{nN})$ and (average) force/radius $(\mathrm{mN} / \mathrm{m})$ vs distance $(\mathrm{nm})$ curves measured on approach using a sulfate-functionalized probe tip on a sulfate-functional ized substrate in $0.0001-0.1 \mathrm{M} \mathrm{NaCl}$ solutions at $\mathrm{pH}$ 5.6. Since the $\mathrm{pK}_{\mathrm{a}}$ of the sulfate group is $\sim 2-2.5^{17}$ and that of the carboxyl group is $3.5-4,{ }^{18}$ both groups are negatively charged. Far from the surface, the cantilever remained undeflected and the net force was zero. At lower ionic strength, the probe tip was deflected upward beginning at approximately $\mathrm{D}<75 \mathrm{~nm}$, consistent with long-range electrical double layer repulsion forces arising from the local ionic osmotic pressure and Maxwell electric stress. ${ }^{19}$ The maximum magnitude of these repulsive forces was measured to be $\sim 0.25 \mathrm{nN}$ (force/radius $2.4 \mathrm{mN} / \mathrm{m}$ ). Even after acquisition of $>50$ force curves from the same surface site, no significant difference was observed in the data, which suggests that there was no irreversible degradation of the surface functional groups or blunting of the probe tip. At $\mathrm{D} \leq 15 \mathrm{~nm}$, a mechanical instability of the cantilever resulted in an abrupt "jump-to-contact" of the probe tip to the surface, due to the gradient of the attractive force exceeding the cantilever spring constant. In this instability region, all accurate data is lost and the slope of this line is equal to the cantilever spring constant, $\mathrm{dF} / \mathrm{dD}=\mathrm{k}_{\mathrm{c}}$. The (average) jump-in distances were found to be $4.7 \mathrm{~nm}(0.0001 \mathrm{M}), 2.03 \mathrm{~nm}$ (0.001 M), no jump-to-contact (0.01 M), and $5 \mathrm{~nm}(0.1$ M). After surface contact was made, the "constant compliance regime" was reached (set to $D=0$ ); here, little or no deformation of the sample took place and the cantilever and sample moved together in unison. With increasing ionic strength, the magnitude and range of the repulsion force decreased due to screening of electrostatic double layer. At $0.1 \mathrm{M} \mathrm{NaCl}$, the net interaction was dominated by van der Waals forces, and the measured force was attractive over the entire distance range with the minimum force value of $63 \mathrm{pN}$ being a measure of the total surface adhesion. The longrange electrostatic forces in experiments using the sulfate-modified probe tip on the sulfate-modified surface were nonhysteretic; i.e., the approach and retract curves overlapped, while the shorter range van der Waals attraction did exhibit hysteresis and increased in magnitude on retraction (data not shown). Similar results have been reported in the literature for 1,2 mercaptoethanesul fonate $\left(\mathrm{HS}\left(\mathrm{CH}_{2}\right) \cdot 2 \mathrm{SO}_{3}{ }^{-}\right)$in pure water, ${ }^{20}$ 11-mercaptoundecanoic acid $\left(\mathrm{HS}\left(\mathrm{CH}_{2}\right)_{10} \mathrm{COOH}\right)$ as a function of ionic strength, ${ }^{20,21}$ 16-thiohexadecanoic acid (16-mercaptohexadecanoic acid) $\left(\mathrm{HS}\left(\mathrm{CH}_{2}\right)_{15} \mathrm{COOH}\right), 16-$ thiohexadecane $\left(\mathrm{HS}\left(\mathrm{CH}_{2}\right)_{13} \mathrm{CH}_{3}\right)$, 16-thiohexadecanol $\left(\mathrm{HS}\left(\mathrm{CH}_{2}\right)_{16} \mathrm{OH}\right)$ monolayers on gold in pure water, ${ }^{22}$ and 11-mercaptoundecanol $\left(\mathrm{HS}\left(\mathrm{CH}_{2}\right)_{11} \mathrm{OH}\right)$ and 16-thiohexadecanoic acid in phosphate buffer (IS $=0.01 \mathrm{M}, \mathrm{pH}=$ 7). 23

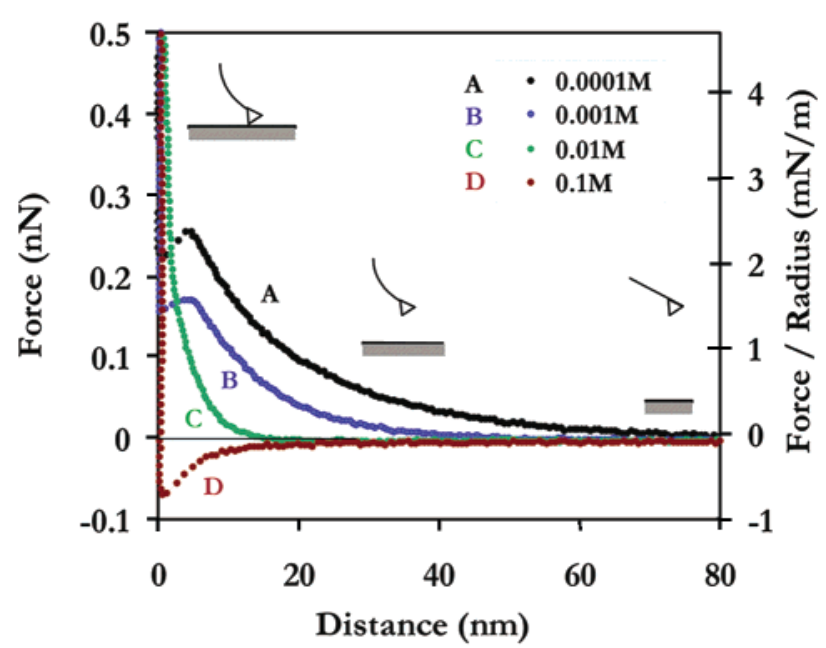

(a)

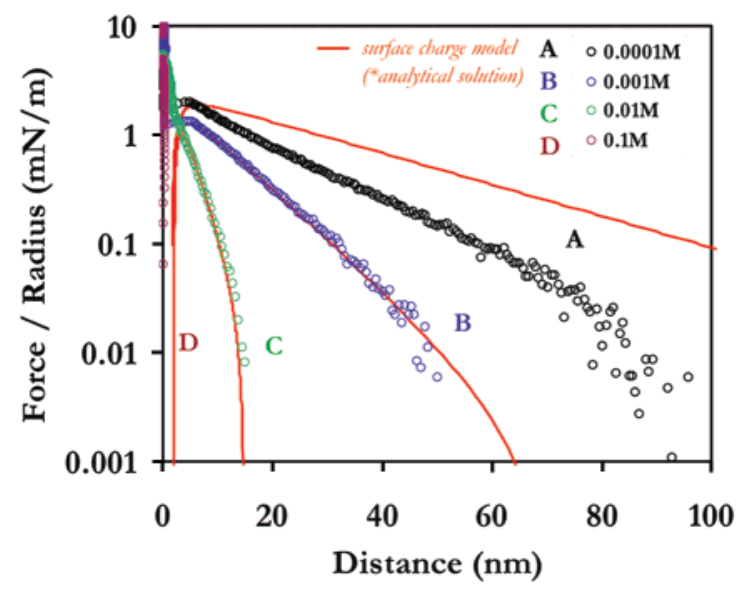

(b)

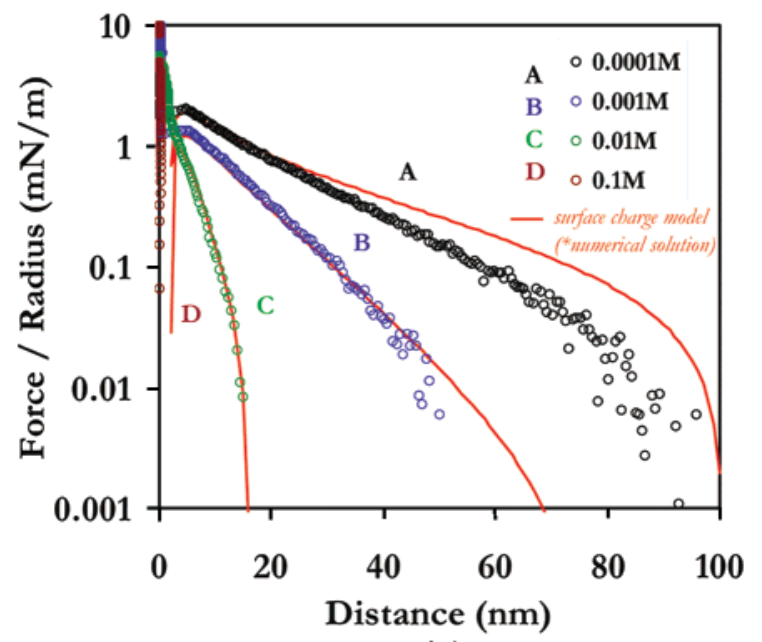

(c)

Figure 5. HRFS data measured on approach with the MFP between sulfate-functionalized probe tip and sulfate-functionalized surface as a function of ionic strength (IS $=0.1,0.01$, 0.001 , and $0.0001 \mathrm{M}$ ) at $\mathrm{pH} \approx 5.6$ : (a) (average) force $(\mathrm{nN})$ and (average) force/radius ( $\mathrm{mN} / \mathrm{m}$ ) vs distance ( $\mathrm{nm})$; (b) (average) force/radius ( $\mathrm{mN} / \mathrm{m}$ ) (logarithmic scale) vs distance $(\mathrm{nm})$ compared to surface charge model (red solid lines, analytical solution, eq A1.6 and Appendix 1.A, using the fitting parameters given in Table 1); (c) (average) force/radius (mN/m) (logarithmic scale) vs distance ( $\mathrm{nm}$ ) compared to surface charge model (red solid lines, nonlinear numerical solution, Appendix $1 B$ ) using the fitting parameters given in Table 1. 
Table 1. Parameters Used in the Theoretical Models of Sulfate-F unctionalized Probe Tip vs Sulfate-Functionalized Surface HRFS Experimental Data ${ }^{a}$

\begin{tabular}{ccccc}
\hline $\begin{array}{c}\text { ionic } \\
\text { strength }\end{array}$ & $\begin{array}{c}\mathrm{R}_{\text {tip }}(\mathrm{nm}) \text { fixed, } \\
\text { measure by SEM } \\
\text { (both models) }\end{array}$ & $\begin{array}{c}\kappa^{-1}(\mathrm{~nm}) \text { fixed, } \\
\text { eq A1.4 } \\
\text { (both models) }\end{array}$ & $\begin{array}{c}\sigma_{1}=\sigma_{2}\left(\mathrm{C} / \mathrm{m}^{2}\right) \text { free, } \\
\text { linear PB: analytical } \\
\text { solution: eq A1.6 }\end{array}$ & $\begin{array}{c}\sigma_{1}=\sigma_{2}\left(\mathrm{C} / \mathrm{m}^{2}\right) \text { free, } \\
\text { nonlinear PB: numerical } \\
\text { solution }\end{array}$ \\
\hline 0.0001 & 127 & 30.0 & $-2.1 \times 10^{-3}$ & $-3.0 \times 10^{-3}$ \\
0.001 & 127 & 9.5 & $-2.2 \times 10^{-3}$ & $-4.0 \times 10^{-3}$ \\
0.01 & 127 & 3.0 & $-3.2 \times 10^{-3}$ & $-15.4 \times 10^{-3}$
\end{tabular}

a Fits included the van der Waals interaction (fixed = parameter fixed to known values; free = fitting parameter allowed to vary in fitting routine).

(a) Comparison of HRF S Data with Theory. The data of Figure 5a were compared to predictions based on the Derjaguin-Landau-Verwey-Overbeek (DLVO) theory. ${ }^{24,25}$ The total interaction force should be a linear summation of attractive van der Waals, repulsive el ectrostatic double layer, and hydrophilic "hydration" forces:

$$
F_{\text {total }}(D)=F_{\text {VDW }}(D)+F_{\text {electrostatic }}(D)+F_{\text {hydration }}(D)
$$

(i) Hydration or Structural Component. A very short range ( $<4 \mathrm{~nm}$ ) monotonic, exponentially repulsive force has been observed experimentally between a variety of different smooth hydrophilic charged surfaces in electrolyte solutions of intermediate and high ionic strength. ${ }^{26}$ The determination of the origin of this force has been quite controversial and is generally attributed to the displacement of hydrated, adsorbed surface counterions and is due to the energy needed to dehydrate the bound counterions, which retain some of their water of hydration on binding. Presumably, this force is highly sensitive to and will decrease with increasing surface roughness. ${ }^{26}$

(ii) van der Waals Component. The nonretarded van der Waals component of the total interaction force was estimated by fitting the highest ionic strength data $(0.1 \mathrm{M})$ to two analytical expressions between 7 and 20 $\mathrm{nm}$ (this distance range is greater than the jump-tocontact cantilever instability region, which was determined as the region where the slope was equal to the cantilever spring constant, but still less than the distances where retardation effects begin to take effect). The first expression was the inverse square power law eq 5 derived using the "Derjaguin approximation":27

$$
F_{\mathrm{VDW}}(D)=-\frac{A R}{6 D^{2}}
$$

where $F$ is the force between a sphere of radius $R$ (assumed to be the probe tip radius, $R_{\text {tip }}=127 \mathrm{~nm}$ ) and a planar surface at separation $D$, and $A$ is the nonretarded Hamaker constant. The second analytical expression was derived using a more accurate surface element integration (SEI) approach by Bhattacharjee et al. ${ }^{28}$

$F_{V D W}(D)=\frac{d\left[-\left(\frac{A}{6}\right)\left[\frac{R}{D}+\frac{R}{D+2 R}+L N\left(\frac{D}{D+2 R}\right)\right]\right]}{d D}$

A Hamaker constant of $5.2 \times 10^{-20} \mathrm{~J}$ was obtained from the fit to eq 5 and one of $35 \times 10^{-20} \mathrm{~J}$ from the fit to eq 6 . These values are close to that predicted using the Lifshitz theory for gol d/ $\mathrm{H}_{2} \mathrm{O} / \mathrm{gol} d$ at $\mathrm{A}=(9-30) \times 10^{-20}$ J (computed from spectroscopic data for gol d surfaces). ${ }^{29}$ Experimental values have been reported from HRFS data computed using eq 5 for gold $/ \mathrm{H}_{2} \mathrm{O} /$ gold at $\mathrm{A}=25$ $\times 10^{-20} \mathrm{~J}^{30}$ and at $\mathrm{A}=10 \times 10^{-20} \mathrm{~J}, 20 \mathrm{CH}_{3}\left(\mathrm{CH}_{2}\right)_{15} \mathrm{OH} /$ $\mathrm{H}_{2} \mathrm{O} / \mathrm{CH}_{3}\left(\mathrm{CH}_{2}\right)_{15} \mathrm{OH}$ at $\mathrm{A}=3.4 \times 10^{-20},{ }^{22}$ and $\mathrm{CH}_{3}\left(\mathrm{CH}_{2}\right)_{10} \mathrm{OH} / \mathrm{H}_{2} \mathrm{O} / \mathrm{CH}_{3}\left(\mathrm{CH}_{2}\right)_{10} \mathrm{OH}$ at $\mathrm{A}=10 \times 10^{-20}$ J 22 and at $\mathrm{A}=4 \times 10^{-20} \mathrm{~J} .20$ These results suggest that the gold substrate dispersion interaction dominates over the surface hydrocarbon layer (i.e., only at very short distances $(D<1 \mathrm{~nm})$ will the less polarizable hydrocarbon layer contribute to the effective Hamaker constant), and hence, we do not resort to a more complicated multilayer model for the effective Hamaker constant. ${ }^{23,30}$ It has also been postulated that smaller probe tip radii allow penetration through the SAM layer, which in this case is approximately $1.6 \mathrm{~nm}$ thick; such a displacement of the hydrocarbon chains upon contact would allow the van der Waals interaction between the underlying gold surfaces to dominate. ${ }^{30}$

(iii) E lectrostatic Double Layer Component. The electrostatic double layer component of the total interaction force was modeled, first, using a constant surface charge density approximation based on the PoissonBoltzmann (PB) equation in which the substrate is assumed to be a plane of constant charge density $\left(\sigma_{1}\right)$ and the probe tip is modeled as a hemisphere of radius,

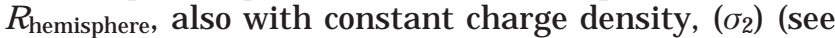
Appendix 1 parts $A$ and $B$ ). Two solutions of the PB equation were obtained and fit to the experimental data shown in Figure 5a: (1) an approximate analytical solution using the linearized PB equation (Appendix $1 \mathrm{~A}$, eq A1.631-33) and (2) a more exact numerical solution using the nonlinear PB equation (Appendix 1B, eq A1. $7^{34}$ )

(b) Summary of Theoretical Results. The results for the constant surface charge model are given in Figure $5 b$ (the analytical solution of eq A1.6) and Figure $5 c$ (the more exact numerical solution), also taking into account the van der Waals force. In both solutions, $\kappa^{-1}$ (calculated from eq A1.4) and $R_{\text {tip }}$ (measured experimentally by SEM) were fixed to their known values, and $\sigma_{1}=\sigma_{2}$ was allowed to be a free fitting parameter. The parameters employed for both models are summarized and compared in Table 1. The value of $\sigma_{1}$ suggests that mercaptoethanesulfonic acid did not have enough carbon to form a densely packed crystalline surface. ${ }^{35}$ The $0.1 \mathrm{M}$ data were assumed to be dominated by van der Waals interactions and, indeed, including or excluding the electrostatic component of the force changed the Hamaker constant obtained by a negligible amount. For the intermediate ionic strength conditions $(0.001$ and $0.01 \mathrm{M}$ ), the nonlinear numerical solution to the surface charge model produced an excellent fit through most of the distance range probed, while the analytical solution increasingly underestimated the force for $D<15 \mathrm{~nm}$. At the lowest ionic strength value $(=0.0001 \mathrm{M})$, the analytical solution could not fit the data over any distance range probed and overestimated the experimental data throughout most of the distance range 
probed, while the full nonlinear numerical solution improved the fit somewhat for $\mathrm{D}<25 \mathrm{~nm}$. The magnitude of the surface charge density calculated from the analytical solution was $0.35-0.5$ times that of the nonlinear solution. I nterestingly, both models predicted an increase in the surface charge density with increasing ionic strength.

Interaction between Sulfate-F unctionalized Probe Tip and CS-GAG-F unctionalized Substrate as a F unction of I onic Strength. The (average) force $(\mathrm{nN})$, (average) force/radius $(\mathrm{mN} / \mathrm{m})$, and (average) stress (MPa) vs distance $(\mathrm{nm})$ between a sulfate-modified probetip and a CS-GAG-modified surface is shown in Figure 6a for ionic strengths ranging from 0.0001 to $1.0 \mathrm{M}$ at pH 5.6. The stress was estimated as the force normalized to the probe tip area (approximated as a hemisphere). Similar to the trends of Figure 5, longrange el ectrostatic repulsive forces were observed which decreased in the magnitude and range with increasing ionic strength. For the lowest ionic strength condition $(0.0001 \mathrm{M} \mathrm{NaCl})$, the repulsive force began at $\mathrm{D} \sim 175$ $\mathrm{nm} \gg \mathrm{L}_{\text {contour }}=35 \mathrm{~nm}$, and reached a maximum magnitude of $\sim 2.5 \mathrm{nN}(\sim 50 \mathrm{mN} / \mathrm{m})$, i.e., much greater than the maximum magnitude of the force reached in the sulfate vs sulfate control experiments at this same IS (i.e., $F_{\max } \sim 2.4 \mathrm{mN} / \mathrm{m}$ ). For the highest ionic strength conditions of 0.1 and $1 \mathrm{M} \mathrm{NaCl}$, the repulsive forces began at $\mathrm{D}<\mathrm{L}_{\text {contour }}=35 \mathrm{~nm}$ and reached maximum magnitudes of $\sim 1 \mathrm{nN}(\sim 20 \mathrm{mN} / \mathrm{m})$ and $\sim 0.5 \mathrm{nN}(\sim 10$ $\mathrm{mN} / \mathrm{m})$, respectively. The sulfate-CS-GAG net interaction force was purely repulsive at all ionic strengths over the entire distance range probed. No jump-to-contact of the cantilever due to van der Waals attraction could be observed when the sulfate probe tip made contact with the top of the CS-GAG brush (as observed previously for poly(L-glutamic acid) brushes ${ }^{36}$ or for sulfate probeunderlying gold substrate interaction as we observed for the sulfate vs sulfate data) at any ionic strength value tested. The measured force per unit CS-chain area was on the order of $\sim 0.1-0.5 \mathrm{MPa}$, which scales to the known macroscopic swelling pressures of CS-GAG chains in vivo. ${ }^{1,37}$ The HRFS experiments using the sulfate-modified probe tip on the CS-GAG-modified surface were nonhysteretic throughout the entire distance range probed, as shown in Figure $6 \mathrm{~b}$, which shows a typical force $(\mathrm{nN})$ vs distance $(\mathrm{nm})$ plot on approach and retract for an individual HRFS experiment at $0.0001 \mathrm{M}$ and $\mathrm{pH}$ 5.6. The standard deviation of each force profile was less than $20 \mathrm{pN}$ and the force profile with standard deviation at $0.1 \mathrm{M}$ is shown in Figure $6 c$.

(a) Comparison of HRFS Data to Theory. The total interaction force between a sulfate-functionalized probe tip and CS-GAG-functionalized surface has an additional steric term compared to eq 4

$$
\begin{array}{r}
F_{\text {total }}(D)=F_{\text {VDW }}(D)+F_{\text {electrostatic }}(D)+ \\
F_{\text {hydration }}(D)+F_{\text {steric }}(D)
\end{array}
$$

where $F_{\text {steric }}(D)$ is the "overlap" repulsion due to deformation of the polymer layer or brush in compression. For flexible polymer chains, $F_{\text {steric }}(D)$ has contributions from configurational entropy and osmotic pressure due to a local increase in chain segment concentration at the interface. ${ }^{4} \mathrm{~F}$ or rigid, rodlike chains, $\mathrm{F}_{\text {steric }}(D)$ includes bending and translational entropy penalties(described in more detail bel ow). ${ }^{38}$ No matter what the molecular mechanism, steric repulsion becomes significant when the probe tip comes in physical contact with the top of the polymer layer or brush, $L_{0}(=$ brush height), which has a maximum value of $L_{\text {contour }}=35 \mathrm{~nm}$ for the case of the CS-GAG-functionalized substrate. The additivity of steric and DLVO forces has been used extensively in the literature ${ }^{39-43}$ but is questioned by others. ${ }^{44,45}$ It is clear that these two components are interrelated and highly dependent on each other. Since steric repulsion, attractive van der Waals forces, intermolecular electrostatic repulsion between neighboring CS-GAG chains on the surface, and hydration repulsion all come into play at shorter distance ranges, ${ }^{46-48}$ the long-range portion of the data $(D>10 \mathrm{~nm})$ was fitted to the purely electrostatic double layer theories. Only the experiments conducted at the higher ionic strength values $(0.01,0.1$, and $1 \mathrm{M}$ ) were compared to theory (for reasons described below). Hence, the data of Figure 6a were compared to the predictions of (1) the surface charge density model using the nonlinear PB equation solved numerically and (2) a model in which the CSGAG brush layer was approximated as a smoothed volume of known fixed charge density (Appendix 1C). 39 The latter model represents the chemically functionalized probe tip as a smooth hemisphere with constant surface charge density, $\sigma_{1}$, and the CS-GAG-functionalized substrate as having a uniform volume charge density, $\rho_{\mathrm{fix}}=-5.9 \times 10^{6} \mathrm{C} / \mathrm{m}^{3}(0.061 \mathrm{M})$. This volume charge density was calculated from the density of CSGAG obtained from scintillation counting ( 0.024 chains/ $\mathrm{nm}^{2}$ ) and the number of charge groups per CS-GAG chain ( 2 charges per disaccharide, 25 disaccharides per chain). This model predicts different nanomechanical behavior in two different distance regimes: region I outside the fixed volume charge region where the PB equation has the same form as the surface charge model, and region II inside the fixed volume charge region where the PB equation has an extra term added to account for $\rho_{\mathrm{fix}}$.

(b) Summary of Theoretical Data Fit Results. The results of fitting the above theoretical models to the data of Figure $6 a$ are given in Figure $6 d$, including the van der Waals interaction. $\kappa^{-1}$ (calculated from eq A1.4), $\mathrm{R}_{\text {tip }}$ (measured experimentally via SEM), $\sigma_{1}$ (taken from theoretical fits of sulfate vs sulfate HRFS data, Table 1 ), and $\rho_{\mathrm{fix}}$ (calculated from scintillation counting data) were fixed to their known values, so that the surface charge model had no free fitting parameters and the volume charge model had one free fitting parameter, $h$, the height of the volume charge (Figure 10). The parameters employed in both models are summarized and compared in Table 2. As expected, the surface charge model greatly underestimated the experimental data throughout the entire distance range probed, while the volume charge model fit better to the data for $\mathrm{D}<$ $25 \mathrm{~nm}$. F or D $>25 \mathrm{~nm}$, the force predicted by the volume charge model decreased dramatically, further underestimating the data with increasing separation distance.

Interaction between Sulfate-Functionalized Probe Tip and CS-GAG-F unctionalized Substrate as a Function of $\mathbf{p H}$. The average force $(\mathrm{nN})$ vs distance $(\mathrm{nm})$ between a sulfate-modified tip and a CSGAG-modified surface for $0.015 \mathrm{M}$ ionic strength at $\mathrm{pH}$ 3.0 and 7.0 is shown in Figure 7. At all distances $D$, the repulsion force at $\mathrm{pH} 7$ was higher than that at $\mathrm{pH} 3$. Since the CS-GAG carboxylic groups have a $\mathrm{pK}_{\mathrm{a}} \sim 3.5-$ $4,{ }^{18}$ al most half the CS-GAG charge would be neutral- 


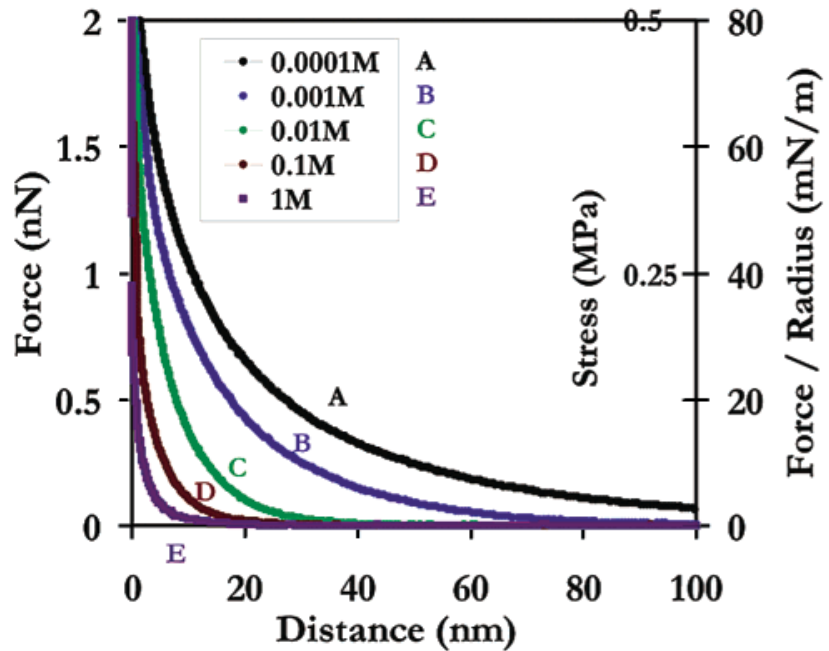

(a)

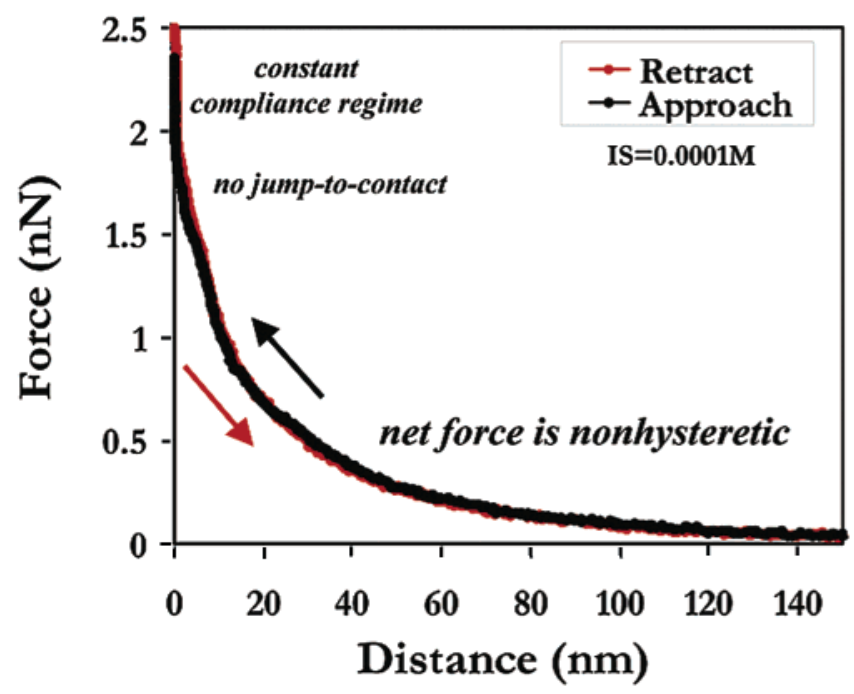

(b)

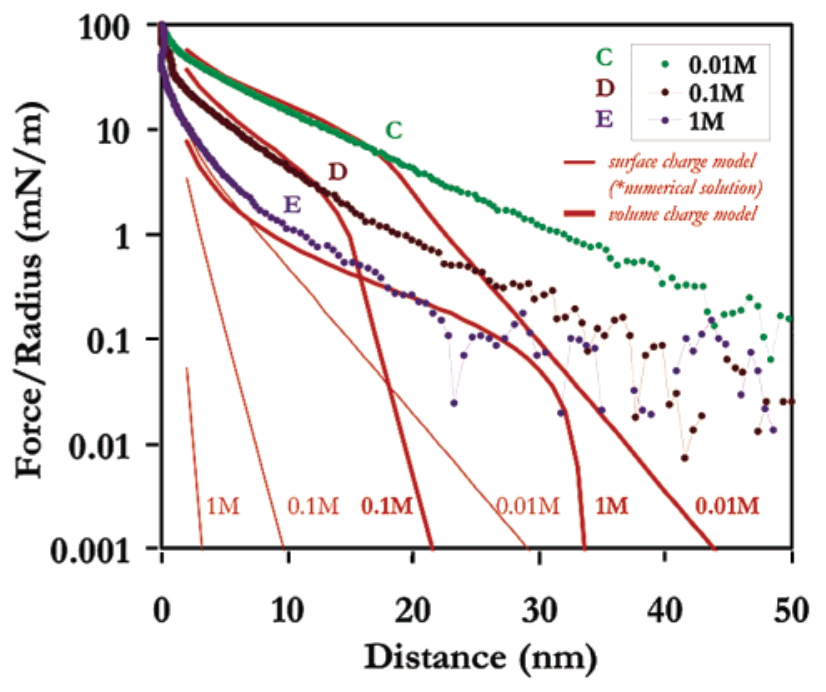

(d)

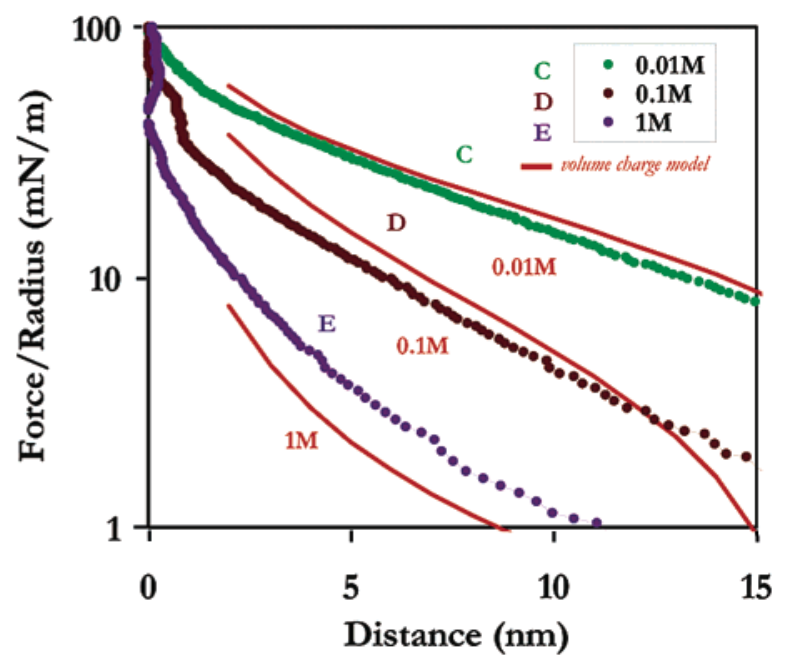

(e)

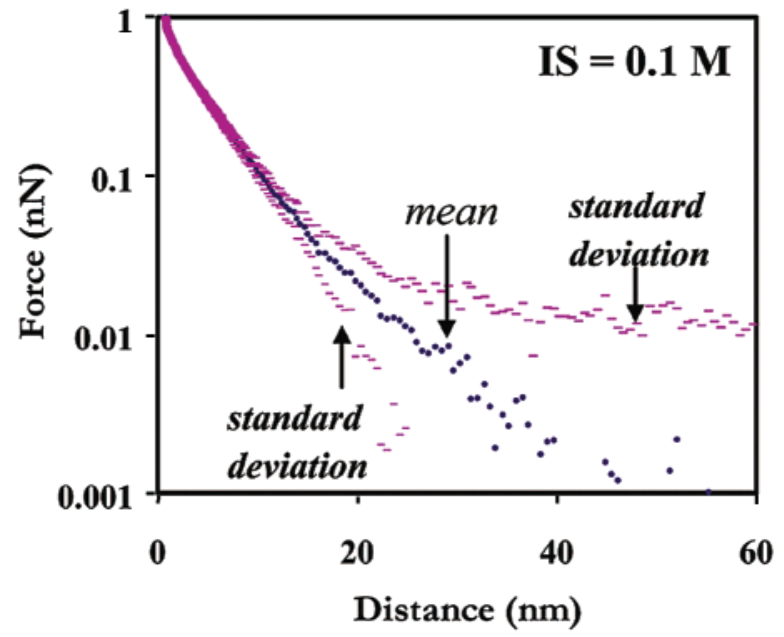

(c)

Figure 6. (a) HRFS data measured on approach with the MFP between sulfate-functionalized probe tip and CS-GAGfunctionalized surface as a function of ionic strength ( $\mathrm{IS}=1,0.1,0.01,0.001$, and $0.0001 \mathrm{M})$ at $\mathrm{pH} \approx 5.6$ : (average) force $(\mathrm{nN})$, (average) force/radius $(\mathrm{mN} / \mathrm{m})$, and stress $(\mathrm{MPa})$ vs distance $(\mathrm{nm})$. (b) Force $(\mathrm{nN})$ vs distance $(\mathrm{nm})$ on approach and retract for an individual force spectroscopy experiment, $\mathrm{pH} \approx 5.6$ and IS $=0.0001 \mathrm{M}$ between sulfate-functionalized probe tip and CS-GAGfunctionalized surface. (c) Force $(\mathrm{nN})$ (logarithmic scale) vs distance $(\mathrm{nm})$ with standard deviation measured at $0.1 \mathrm{M}$ at pH $\approx$ 5.6. (d) (Average) force/radius ( $\mathrm{mN} / \mathrm{m}$ ) (logarithmic scale) vs distance $(\mathrm{nm})$ compared to surface charge model (thin red solid lines, nonlinear numerical solution, Appendix 1B) and volume charge model (thick red solid lines, Appendix 1C using the fitting parameters given in Table 2). (e) Expanded plot of Figure 6d, (average) force/radius (mN/m) (logarithmic scale) vs distance (nm), compared to volume charge model (thick red solid lines). 
Table 2. Parameters Used in the Theoretical Models of Sulfate-F unctionalized Tip vs CS-GAG-Functionalized Surface HRFS Experimental Data

\begin{tabular}{|c|c|c|c|c|c|}
\hline $\begin{array}{l}\text { ionic } \\
\text { strength } \\
(\mathrm{M} \mathrm{NaCl})\end{array}$ & $\begin{array}{c}\kappa^{-1}(\mathrm{~nm}) \text { fixed, } \\
\text { eq A1.4 } \\
\text { (both models) }\end{array}$ & $\begin{array}{l}\mathrm{R}_{\text {tip }}(\mathrm{nm}) \text { fixed, } \\
\text { measured by SEM } \\
\text { (both models) }\end{array}$ & $\begin{array}{c}\sigma_{1}\left(\mathrm{C} / \mathrm{m}^{2}\right) \text { fixed, } \\
\text { nonlinear } \mathrm{PB} \\
\text { numerical solution }\end{array}$ & $\begin{array}{c}\sigma_{2}\left(\mathrm{C} / \mathrm{m}^{2}\right)=\rho_{\mathrm{fix}} \times \mathrm{h} \text { fixed, } \\
\text { measured by } \\
\text { scintillation counting }\end{array}$ & $\begin{array}{c}\text { h (nm) free, } \\
\text { volume charge } \\
\text { height }\end{array}$ \\
\hline 0.01 & 3.0 & 25 & $-15 \times 10^{-3}$ & -0.19 & 17.6 \\
\hline 0.1 & 0.95 & 25 & $-15 \times 10^{-3}$ & -0.19 & 14.1 \\
\hline 1 & 0.3 & 25 & $-15 \times 10^{-3}$ & -0.19 & 31.8 \\
\hline
\end{tabular}

a Fixed = parameter fixed to known values; free = fitting parameter allowed to vary in fitting routine)

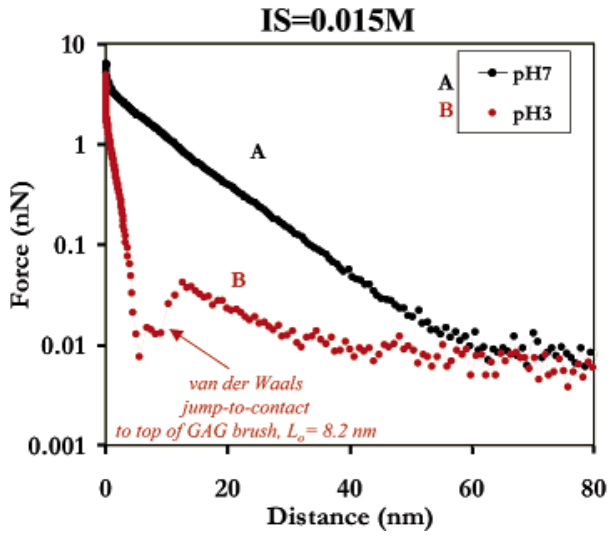

Figure 7. Comparison of (average) force/radius $(\mathrm{mN} / \mathrm{m})$ (logarithmic scale) vs distance (nm) curves measured on approach between sulfate-modified tip and CS-GAG-functionalized surface at $\mathrm{pH}=3$ and $\mathrm{pH}=7(\mathrm{IS}=0.015 \mathrm{M})$.

ized at $\mathrm{pH} \mathrm{3,consistent} \mathrm{with} \mathrm{the} \mathrm{lower} \mathrm{force} \mathrm{that} \mathrm{was}$ observed. Thus, the data in Figure 7 represents the effect of varying the CS-GAG charge at nearly constant ionic strength, in contrast to the data of Figure 6, which represents the effects of varying the CS-GAG surface potential at nearly constant charge (via changes in ionic strength at constant $\mathrm{pH}$ ). Interestingly, for the less charged low pH conditions, one can observe the van der Waals jump-to-contact to the top of CS-GAG brush at a separation distance of $8.2 \mathrm{~nm}$.

Interaction between Hydroxy-Functionalized Probe Tip and CS-GAG-F unctionalized Substrate as a F unction of I onic Strength. The (average) force $(\mathrm{nN})$, (average) force/radius $(\mathrm{mN} / \mathrm{m})$, and (average) stress (MPa) vs distance (nm) between a hydroxymodified probe tip and a CS-GAG-modified surface are shown in Figure $8 a$ for ionic strengths ranging from 0.0001 to $3.0 \mathrm{M}$ at pH 5.6 (y-axis on a linear scale) and Figure 8b ((average) force/radius $(\mathrm{mN} / \mathrm{m})$ y-axis logarithmic scale). The neutral, hydroxy-functionalized probe tip is useful because it does not exhibit a pH-dependent change in ionization and is hydrophilic. One can clearly observe that, even for this case of the so-called neutral probe tip vs a negatively charged CS-GAG surface, long-range el ectrostatic repul si ve forces are still present up to $D=200 \mathrm{~nm}$ at the lowest ionic strength conditions, $0.0001 \mathrm{M}$. One possible origin of this el ectrostatic repulsive force is the nonspecific adsorption of counterions from the solution to the probe tip, which causes the probe tip to have a nonzero effective surface charge. Figure 9 shows the (average) force/radius $(\mathrm{mN} / \mathrm{m})$ on a logarithmic scale vs distance (nm) for the hydroxyfunctionalized compared to the sulfate-functionalized probe tips vs CS-GAG-modified surface at IS $=0.1 \mathrm{M}$, $\mathrm{pH} \sim 5.6$. It was observed that the force for the hydroxyfunctionalized tip was always less than that for the sulfate-functionalized probe tip throughout the entire distance range measured.

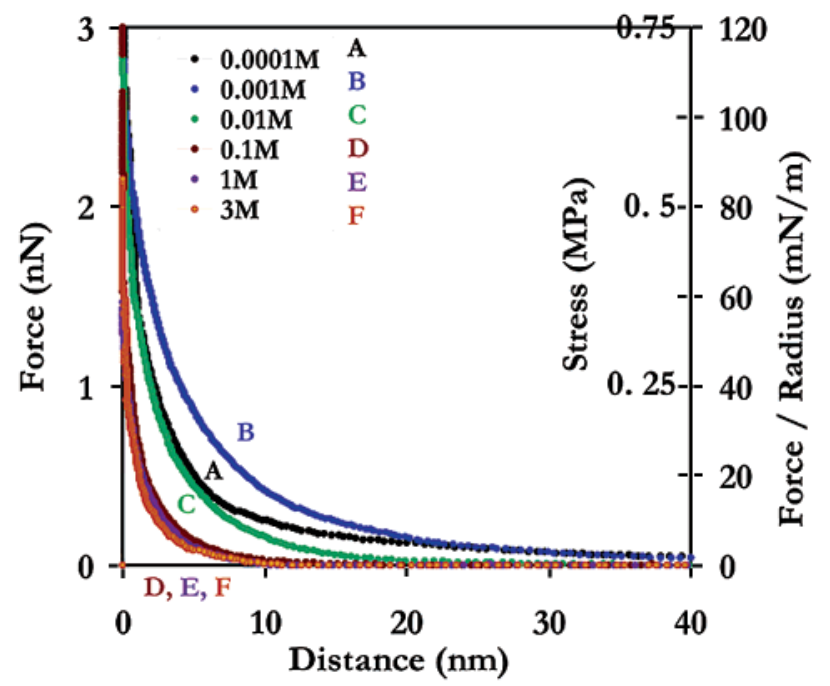

(a)

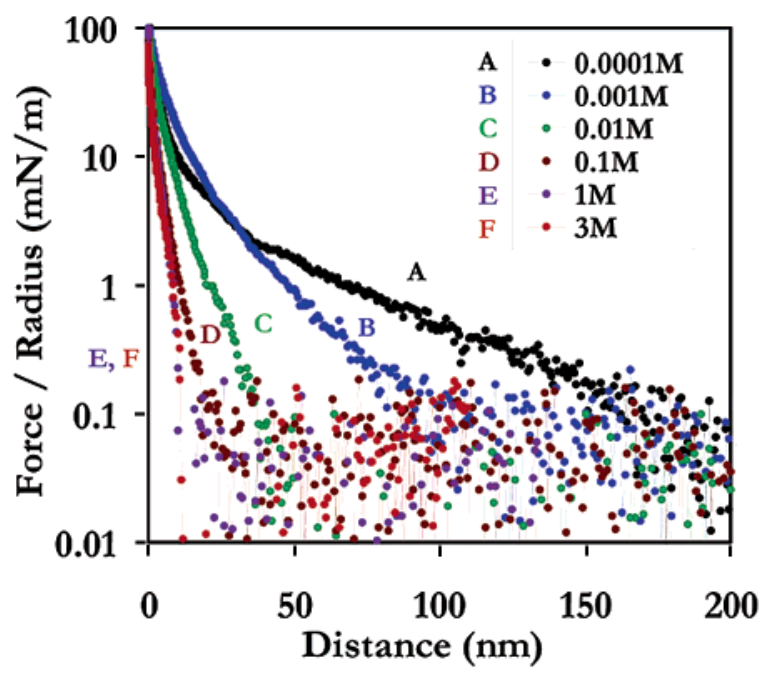

(b)

Figure 8. HRFS data measured on approach with the MFP between hydroxy-functionalized probe tip and CS-GAGfunctionalized surface as a function of ionic strength (IS $=3$, $1,0.1,0.01,0.001$, and $0.0001 \mathrm{M}$ ) at $\mathrm{pH} \approx 5.6$ : (a) (average) force $(\mathrm{nN})$, (average) force/radius $(\mathrm{mN} / \mathrm{m})$, and stress (MPa) vs distance (nm); (b) (average) force/radius $(\mathrm{mN} / \mathrm{m})$ (logarithmic scale) vs distance $(\mathrm{nm})$.

\section{Discussion}

In this study, we prepared highly purified and wellcharacterized chemically end-grafted polymer brush surfaces of negatively charged CS-GAG macromolecules, and we report the first molecular mechanics measurements on this system using the technique of high-resolution force spectroscopy. A combined experimental and theoretical approach was aimed at understanding the contributions of the four constituent intermolecular interactions (eq 7) to the total measured force between the brush layer and probe tip. 


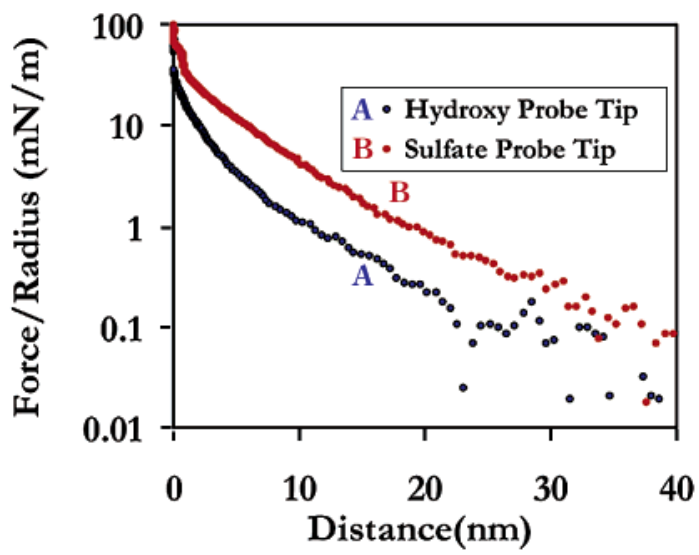

Figure 9. Comparison HRFS data measured on approach for sulfate-modified probe tip vs CS-GAG-functionalized surface and hydroxy-modified probe tip vs CS-GAG-functionalized surface both at $\mathrm{pH} \approx 5.6$ and IS $=0.1 \mathrm{M}$ : (average) force/ radius $(\mathrm{mN} / \mathrm{m})$ (logarithmic scale) vs distance $(\mathrm{nm})$

Control Experiments: Interaction between Sulfate-Functionalized Probe Tip and SulfateFunctionalized Substrate as a Function of Ionic Strength. To interpret our CS-GAG brush measurements, the sulfate vs sulfate control data of Figure 5 provided two important pieces of information: (1) an estimate of the sulfate charge density of the probe tip and (2) a control to investigate the validity of frequently used theoretical models to represent interaction forces in this relatively simpler system. The measurement of repulsive DLVO forces between nonpolymeric charged surfaces is well documented in the literature using both the surface force apparatus (and similar instruments) ${ }^{46-51}$ and the atomic force microscope. ${ }^{20-23,30,52-62}$

All of the theoretical models presented in this paper employ constant surface charge boundary conditions, rather than constant potential, for two reasons. First, both the substrate and the tip were el ectrically isolated from each other and from the instrument ground and, therefore, not electrically connected to any source that would maintain them at a constant potential. ${ }^{63}$ Second, theoretical modeling of the ionization state of surface charges in our system suggests that the CS-sulfate ester as well as carboxyl-derived charge is nearly constant over the range of $\mathrm{pH}$ and ionic strength conditions and at all tip-substrate separation distances. In general, the $\mathrm{pH}$ at the charged surface and the ionization state of the surface charge groups will depend on bath $\mathrm{pH}$, bath ionic strength, and the $\mathrm{pK}_{\mathrm{a}}$ 's of the charge groups. A lower bound estimate of the surface $\mathrm{pH}$ was calculated using the linearized PB equation ${ }^{31}$ for our ionic strength range at all distances, given the bath $\mathrm{pH} 5.6$ and the relevant $\mathrm{pK}_{\mathrm{a}}$ 's. The $\mathrm{pH}$ at the surface was calculated to be $>5$, even at the lowest ionic strength, which is much higher than the $\mathrm{pK}_{a}$ 's of the charge groups. Thus, less than $1 \%$ of the charged groups would be neutralized (protonated). In addition, we used a model ${ }^{64}$ to calculate the "charge regulation parameter $\alpha$ " that characterizes approaching charged surfaces as maintaining constant charge or constant potential. From our data, this parameter was calculated to al ways be in the constant charge regime.

The model using eqs A1.6 and A1.7 fit reasonably well to the sulfate-sulfate data of Figure 5, parts $a$ and $b$, respectively, except at short distances $D<15 \mathrm{~nm}$ and at low ionic strength for all distances. The overestimation of the data by the linearized analytical solution (eq
A1.6) at low ionic strength is most likely due to (1) the high values of the potential, which violate the conditions of the linearizing approximation, ${ }^{65}$ and (2) modeling of the tip as an effective hemisphere, while the actual tip geometry is ill-defined and more similar to that of a pyramid. These errors should become increasingly significant at lower ionic strength as the Debye length (the length scale over which electrostatic forces act) approaches $\mathrm{R}_{\text {tip. }}$. This tip size-shape problem is also consistent with the apparent increase in predicted surface charge density with increasing ionic strength (Table 1). For the smaller pyramid shape, the model will tend to overestimate the el ectrostatic force especially at lower ionic strengths. To compensate, the fit values of the surface charge densities will be smaller than the actual values with decreasing ionic strength.

As noted previously, the linearized analytical solution underestimates the experimental data for $D<15 \mathrm{~nm}$. This is due to the fact that in the constant surface charge model, the magnitude of the potential increases as the tip approaches the surface, and eventually exceeds the values appropriate for the linearization approximation (see Appendix 1A).28,66 The linearized solution will then overestimate the data when the tip is far from the surface and underestimate the actual charge density. The nonlinear solution avoids this problem and fits closer to the data (Figure $5 c$ ). Interestingly, one may note the absence of the van der Waals jump-to-contact at $0.01 \mathrm{M}$ data, presumably due to an additional repulsive hydration force.

Interaction between Sulfate-F unctionalized Probe Tip and CS-GAG-F unctionalized Substrate as a F unction of Ionic Strength. Several studies have reported the measurement of repulsive forces between surfaces bearing adsorbed ${ }^{67-71}$ or hydrophobically anchored $^{36,72,73}$ polyelectrolyte layers using both the AFM and the surface forces apparatus (SFA). In contrast, the CS-GAG polyelectrolyte chains in our study were chemically end-grafted to the surface. Adsorbing polymer layers exhibit significant degrees of hysteresis, ${ }^{69,74}$ while the interaction of the CS-GAGs with the underlying substrate is shown here to be minimal as evidenced by the nonhysteric, reversible overlap of approach and retraction curves (Figure 6b). The magnitude of the force was consistent at three different locations and the standard deviation of the experiment at each ionic strength was less than $20 \mathrm{pN}$, which indicated the uniformity of the CS-GAG end-grafted substrate. At low ionic strength, we expect the individual CS-GAGs to be in a more extended, rigid rodlike conformation where the intramolecular repulsion between neighboring charges on the CS-GAG chains outweighs the entropic forces driving the chain to a random coil. As the ionic strength is increased, e.g., to $1 \mathrm{M}$, the chains are expected to collapse into a more random coillike configuration as the intra- and intermolecular electrostatic repulsion is screened by the salt and the Debye length is reduced, ${ }^{75}$ contributing in part to the dramatic decrease in measured repulsive force with increasing ionic strength (Figure 6a). A detailed study is currently underway to investigate the conformation of the CSGAG chains at the surface directly at various ionic strength conditions using the technique of neutron reflectivity ${ }^{76,77}$ and will be reported in a subsequent paper.

Model s were fit to the data of Figure 6, parts $d$ and $e$ at the three highest ionic strengths (0.01, 0.1, and $1 \mathrm{M})$. 
$\mathrm{R}_{\text {tip }}$ for these experiments was $25 \mathrm{~nm}$, smaller than that used for the sulfate-sulfate experiments. The approximation of the tip as a hemisphere can only be used when $\mathrm{R}_{\text {tip }}$ is much greater than the distance over which the force acts. ${ }^{26,28}$ Therefore, it is not appropriate to use the models described in this paper for the lower ionic strengths $(0.001-0.0001 \mathrm{M})$ at which the Debye length is on the order or larger than $\mathrm{R}_{\text {tip }}$ (see Appendix $1 \mathrm{~B}$ ).

(a) Constant Surface Charge Model (Nonlinear Numerical Solution). I certain previous studies, 36,69,72 a flat surface charge model was used as a first approximation to represent polyelectrolyte layers. However, discrepancies between such a model and experimental data would be expected for the following reasons. First, a flat surface charge may not accurately reflect the distributed charge of the polyelectrolyte chains on the surface, even for adsorbed layers, since loops (segments terminally bound with the rest in contact with the solvent), trains (series of consecutively adsorbed segments), and tails (having one end bound and the other dangling in solution) are typically present. ${ }^{78}$ Second, for $D<L_{o}$ (where $L_{o}=$ the brush height), steric forces are present. For neutral end-grafted polymer brushes, these forces are due to an attractive elastic restoring force due to configurational entropy and a repulsive osmotic pressure. ${ }^{4}$ The osmotic pressure originates from solvent translational entropy and intrachain excluded volume due to short-range monomer-solvent affinity and/or monomer-monomer repulsion. U pon compression and confinement, the local increase in chain segment concentration at the interface leads to an increase in the osmotic pressure and a decrease in the elastic restoring force, with the osmotic contribution dominating, especially at strong compressions. For rigid, rodlike chains, the steric component may also include bending and translational entropy penalties. ${ }^{38}$ The steric component of the force is directly affected by the conformation of the polymer chains on the surface and will change dramatically depending on the electrostatics of the system (i.e., ionic strength, $\mathrm{pH}$, etc.) and the interaction with the substrate (e.g., adsorbing vs nonadsorbing). Third, lateral intermolecular electrostatic repulsion between neighboring polyel ectrolyte chains on the surface may al so play a role for decreasing $D<L_{0}$.

Figure $6 \mathrm{~d}$ compares the flat constant surface charge model pictured in Figure 10a with our CS-GAG brush data. With all of the model parameters fixed to their known values, the model does predict the correct trend with varying ionic strength but, as expected, it severely underestimates the data over all of the distance ranges measured and gives us a good indication of the magnitude of the additional forces due to the polymeric structure of the CS-GAGs as a function of ionic strength. Some of the model parameters (e.g., $\sigma_{1}, \sigma_{2}, \kappa^{-1}$ ) could be varied so that the model more closely fits the data but artificially changing the model parameters with no physical reason would reveal no new information and could be misleading.

(b) Volume Charge Model. Next, we used a "volume charge model" which represents the CS-GAG brush layer as a fixed volume charge density on the substrate (Figure 10b). The height of the volume charge, h, which is equivalent to the brush height, $L_{0}$, was the only free fitting parameter in the model, and considerably improved fits were obtained (Figure 6, parts $d$ and e) compared to the flat constant surface charge model for $D<25 \mathrm{~nm}$ using $\mathrm{h}=\mathrm{L}_{\mathrm{o}}$ values of $17.6 \mathrm{~nm}$ (IS $=0.01$
$M), 14.1 \mathrm{~nm}(\mathrm{IS}=0.1 \mathrm{M})$, and $31.8 \mathrm{~nm}(\mathrm{IS}=1 \mathrm{M})$. The values obtained for $h=L_{0}<L_{\text {contour suggest that the }}$ CS-GAG chains are not fully extended, but not completely collapsed on the surface, even at the highest ionic strength (1 M). The h values and, hence, the magnitude of the purely el ectrostatic forces obtained are most likely overestimated due to the presence of steric and lateral intermolecular electrostatic repulsion which have not yet been included in the model. This is especially true at IS = $1 \mathrm{M}$ since the Debye length is small; the electrostatic forces start when the tip is inside the brush in a region where steric forces are most significant. At shorter distances ( $<15 \mathrm{~nm}$, Figure $6 \mathrm{e}$ ), the model still underestimates the experimental data at $1 \mathrm{M}$ and, as a first approximation, gives an indication of the magnitude of the steric and lateral intermolecular el ectrostatic repulsive components.

For $\mathrm{D}>25 \mathrm{~nm}$, the volume charge model severely underestimates the experimental data. One reason for this discrepancy is the approximation of the probe tip as a hemisphere, as discussed above. Another source of error is the representation of the CS-GAG brush as a volume of uniform charge density. The CS-GAGs are approximately $6 \mathrm{~nm}$ apart and have a nonuniform charge distribution in the space within the brush $(D<$ $L_{o}$ ), which will also affect the ionic double layer distribution within and above the brush $\left(D>L_{0}\right)$.

The vol ume charge model predicts a transition in the force vs distance curve at the position $\mathrm{h}=\mathrm{L}_{\mathrm{o}}$. A sharp transition is not observed in our experimental data, although it has been observed to varying degrees in other polyelectrolyte systems in the literature. $36,68,72$ This may be due to the relatively low grafting density $\left(\Gamma=0.024\right.$ chains $\left./ \mathrm{nm}^{2}\right)$ compared to the systems re ported in the literature (e.g., $\Gamma=0.13-0.41$ chains/ $\left.\mathrm{nm}^{2}\right) .{ }^{36}$ It was noticed that the curve fit of volume charge model extends longer distances at $1 \mathrm{M}$ than $0.1 \mathrm{M}$, which is due to ignoring the steric component in our model. The curve fit was carried out using a volume charge model that includes only the electrostatic component of force at various salt concentrations. When there exists a region of space in which electrostatic forces dominate (i.e., for ionic strengths $\geq 0.1 \mathrm{M}$ ), the model is able to predict a value for the brush height that is not significantly affected by the presence of steric forces, consistent with the assumptions of our model. However, at $1 \mathrm{M}$ salt concentration, the electrostatic interactions are minimized and become on the order of steric interactions on the length scale of the brush layer. Therefore, by fitting the electrostatic volume charge model to the total measured force in this regime, the brush height is overestimated, which results in a predicted force that extends further from the brush at $1 \mathrm{M}$ than $0.1 \mathrm{M}$. It should al so be noted that the volume charge model predicts a significant el ectrostatic repulsive force even at the highest ionic strength of $1 \mathrm{M}, \mathrm{a}$ condition typically thought to screen electrostatic interactions.

Hydroxy-F unctionalized Probe Tip Versus CSGAG-Functionalized Substrate as a Function of I onic Strength. When the hydroxy-functionalized probe tip was used, long-range electrostatic repulsive forces are clearly present up to D $200 \mathrm{~nm}$ in $0.0001 \mathrm{M} \mathrm{NaCl}$, the lowest ionic strength condition. It was postulated that these forces might arise, in part, from an effective surface charge on the probe tip due to nonspecific anion adsorption. In addition, although $\sigma_{1}=0$, the models still 
predict an electrostatic repulsion force due to the geometry of the tip, bath, and substrate system in which the tip surface is impermeable to ions (see Appendix 1A). ${ }^{32}$

\section{Conclusions}

The total intersurface force between CS-GAG brush layers and probe tips of known chemistry $\left(-\mathrm{SO}_{3}{ }^{-},-\mathrm{OH}\right)$ exhibited a long-range ( $\mathrm{D} \leq 175 \mathrm{~nm}$ compared to $\mathrm{L}_{\text {contour }}$ $=35 \mathrm{~nm}$ ), nonlinear, purely repulsive behavior that decreased in magnitude and range with increasing ionic strength, and decreasing $\mathrm{pH}$. At physiological ionic strength and $\mathrm{pH}$, the measured stress (i.e., force normalized to the area per chain) is on the order of that reported previously as the proteoglycan swelling pressure within intact cartilage tissue. F or $\mathrm{D}>\mathrm{L}_{\text {contour, }}$ the inter-surface interaction is dominated by electrostatic double layer forces while for $D<L_{\text {contour, }}$ steric interactions also come into play. At shorter distance ranges, van der Waals and hydration forces may also be present but are expected to be relatively smaller in magnitude. A detailed comparison of CS-GAG-tip interactions with those between the tip and a nonpolymeric surface charge control system $\left(-\mathrm{SO}_{3}{ }^{-}\right.$vs $\left.-\mathrm{SO}_{3}{ }^{-}\right)$was further interpreted using two theoretical electrostatic double layer models based on the Poisson-Boltzmann equation. These results provided an improved understanding of how CS-GAG polymers can sustain compressive loads in macroscopic tissues such as cartilage. Not surprisingly, the CS-GAG brush layer could not be modeled accurately using a constant surface charge model, which severely underestimated the magnitude of the intersurface force throughout the distance range measured. The volume charge model fit better to the experimental data for reasonable values of the brush height (the only fitting parameter), suggesting that the volume distribution of charge on the CS-GAGs leads to a significant increase in the magnitude of the repulsive force, especially for $D<L_{0}$. Future experiments to directly determine the brush height as a function of ionic strength will enable us to verify the validity of these fits and to further quantify the distinct contribution of steric and lateral electrostatic interactions to the total force. Underestimation of the data by the volume charge theory for $D>25 \mathrm{~nm}$ suggests that the true, nonuniform, rodlike charge distribution al ong individual CSGAG chains may be important in understanding the interaction force at these longer range distances.

In summary, the unique nanomechanical equilibrium behavior of the CS-GAG brush layer nonuniform (nonplanar) charge distribution is due in part to lateral intermolecular el ectrostatic interactions and the unique interplay between electrostatic and steric forces. U nder nonequilibrium conditions, e.g., the deformation of cartilage during joint motion in vivo, additional ratedependent and hydrodynamic effects associated with fluid flow through the CS-GAG brush will be important. Ongoing experiments using a range of tip approach velocities are aimed at studying such hydrodynamic effects. In addition, the dependence of the measured force on the precise structure and sequence of charge al ong the CS-GAG chains, which vary with the developmental stage of the tissue and with the onset of diseases (e.g., osteoarthritis) can be studied using the experimental approach described here.

Acknowledgment. The authors thank Professor Paul Laibinis, Ivan Lee, Cheolmin Park, Stella Park, and J iang-Ti Kong for insightful discussions and technical assistance. We thank Richard Michalitsch for help with the gold evaporator, Mallik Sundaram for CSGAG molecular weight analysis, Monica Rixman for AFM images, and J ason Cleveland, Roger Proksch, and Clint Callahan (Asylum Research, Inc.) for excellent technical assistance and advice on issues concerning the MFP. This research was supported by the Dupont-MIT Alliance, NIH Grant AR45779, a Whitaker F oundation fellowship (D.D.), and the Shriners of North America.

\section{Appendix 1. Theoretical Models for Electrostatic Forces: Diffuse Electrical Double Layer Theory}

The Poisson-Boltzmann (PB) equation gives an expression for the el ectrical potential, $\Phi$ (volts), between two charged surfaces in an electrolyte solution which, for a mono-monoval ent el ectrolyte has the form: ${ }^{19,66}$

$$
\nabla^{2} \Phi=\frac{2 \mathscr{F} \mathrm{C}_{0}}{\epsilon_{\mathrm{w}}} \sinh \left(\frac{\mathscr{F} \Phi}{\mathrm{RT}}\right)
$$

where $\mathscr{F}$ is the Faraday constant $(=96500 \mathrm{C} / \mathrm{mol}), \mathrm{C}_{0}$ the bulk concentration of ions $\left(\mathrm{mol} / \mathrm{m}^{3}\right), \epsilon_{\mathrm{w}}$ the diel ectric permittivity of water $\left(=6.9 \times 10^{-10} \mathrm{C} / \mathrm{Nm}^{2}\right), \mathrm{R}$ the universal gas constant $(=8.314 \mathrm{~J} / \mathrm{mol} \cdot \mathrm{K})$, and $\mathrm{T}$ the absolute temperature $=298 \mathrm{~K}$. To uniquely determine the potential, two boundary conditions on either the potential or its derivative (the electric field) are required. Unfortunately, the PB equation is nonlinear and therefore is difficult to solve analytically except for simple geometries. The force, $F$, per unit area acting in the $z$-direction on the charged surface or, more generally, at any position $z=z_{0}$ between the charged surfaces is the sum of two terms: the osmotic pressure due to the ion concentration gradients and the Maxwell electric field stress due to the force of the electric field action on ionic moieties: ${ }^{19,66}$

$$
\frac{\mathrm{F}}{\text { area }}=\left.\left(2 \mathrm{RTC}\left(\cosh \left(\frac{\mathscr{F} \Phi}{\mathrm{RT}}\right)-1\right)+\frac{\epsilon_{\mathrm{w}}}{2}(\nabla \Phi)^{2}\right)\right|_{\mathrm{z}=\mathrm{z}_{0}}
$$

1.A. Constant Surface Charge Density Model: Analytical Solution. ${ }^{26,31-33}$ This model represents the tip as a smooth hemisphere with constant surface charge per unit area, $\sigma_{1}\left(\mathrm{C} / \mathrm{m}^{2}\right)$, and the substrate as a flat plane with constant surface charge per unit area, $\sigma_{2}$. An analytical solution of the linearized PB equation is often used in the literature and can be obtained by first linearizing eq A1.1 for small enough $\mathscr{F} \Phi / R T$ to obtain

$$
\nabla^{2} \Phi \approx \frac{2 \mathscr{F}^{2} C_{0}}{\epsilon_{\mathrm{w}} \mathrm{RT}} \Phi=\kappa^{2} \Phi
$$

where $\kappa^{-1}$ is the electrical Debye length that can be calculated independently from the ionic strength using

$$
\kappa^{-1}=\sqrt{\frac{\epsilon_{\mathrm{w}} \mathrm{RT}}{2 \mathrm{z}^{2} \mathscr{F}^{2} \mathrm{C}_{\mathrm{io}}}}
$$

where the ion valence $z=1$ for our experiments. The PB equation has been solved for two infinite parallel planes of charge, ${ }^{32}$ and the result integrated to obtain the force between a hemisphere and plane. ${ }^{33} \mathrm{~F}$ or two 
infinite planes of charge with the specified surface charges $\sigma_{1}$ and $\sigma_{2}$, the boundary conditions at these surfaces are: $\partial \Phi / \partial z=\sigma_{1} / \epsilon_{\mathrm{w}}$ and $\partial \Phi / \partial z=-\sigma_{2} / \epsilon_{\mathrm{w}}$. As $\mathrm{z} \rightarrow$ $\infty$, the potential and the electric field approach zero. The force per unit area between two infinite flat planes of charge having densities $\sigma_{1}$ and $\sigma_{2}$ and separated by a distance $D$ reduces to $^{32}$

$$
\frac{\mathrm{F}_{\text {flat }}}{\text { area }}=\frac{\sigma_{1}^{2}+2 \sigma_{1} \sigma_{2} \cosh (\kappa \mathrm{D})+\sigma_{2}^{2}}{2 \epsilon_{\mathrm{w}} \sinh ^{2}(\kappa \mathrm{D})}
$$

The force on a hemispherical tip of radius $R_{\text {hemisphere }}$ is obtained by integrating the force between flat surfaces over appropriately sized concentric cylinders (Figure 10a). If the surface charge on the tip and the substrate are of the same order and $\kappa \mathrm{D}$ is small, then the $\sigma^{2}$ terms can be neglected and the sinh can be linearized, thus yiel ding

$$
\mathrm{F}_{\text {hemisphere }} \approx \frac{4 \pi \sigma_{1} \sigma_{2} \mathrm{R}_{\text {hemisphere }}}{\epsilon_{\mathrm{w}} \kappa} \mathrm{e}^{-\kappa \mathrm{D}}
$$

This approximation is only valid when $|\Phi|$ is much smaller than the "thermal voltage" RT/F $\approx 25.7 \mathrm{mV}$. When $|\Phi| \gg 25.7 \mathrm{mV}$, the linearized model will overestimate the force. As we are using a constant charge boundary condition, the magnitude of the potential on the surface will increase as the tip approaches the surface. Therefore, when using a constant charge boundary condition, the linearized PB equation may not be accurate for small separations.

B. Constant Surface Charge Model: Numerical Solution. We used a Newton method on finite differences ${ }^{34}$ to solve the full nonlinear PB equation subject to one boundary condition at each surface. The force between two infinite charged planes was first obtained and then numerically integrated to give the force between a hemispherical tip and planar substrate. Since the problem is one-dimensional, the potential in space can be represented as a one-dimensional matrix or vector in which each entry is the potential at evenly spaced points along the z-direction. The derivatives in the z-direction can be written as differences between neighboring points. The PB equation for each discrete entry plus the boundary conditions give a set of $\mathrm{N}$ nonlinear equations, where $\mathrm{N}$ is the number of discretizations, all satisfied if the potential at each point is correct. If a close enough initial guess for the value of the potential at all points is given, then that guess can be refined using a Taylor series expansion. This is repeated until the change in potential at each step is smaller than an error threshold. This algorithm is known as a Newton method for solving multidimensional systems. The potential is then converted to a force by taking a bounding box with one surface at point $\mathrm{i}$ between the two charged planes (where the derivative of the potential is zero; i.e., the electrical field is zero) and the other surface at infinity (where the potential and el ectric fields are zero). The force on the enclosed surface is then

$$
\frac{\mathrm{F}}{\text { area }}=\left(2 \mathrm{RTC} \mathrm{C}_{0} \cosh \left(\frac{\mathscr{F} \Phi[\mathrm{i}]}{\mathrm{RT}}\right)-1\right)
$$

The hemispherical tip geometry is approximated by using the calculated force between the flat surfaces and summing up the force on appropriately sized concentric cylinders. In effect, this method, sometimes known as surface element integration (SEI), ${ }^{28}$ is the numerical version of the integral for the linearized hemispheretip solution above. SEI will give the exact interaction if the stress (force per unit area) is normal to the surfaces. This requirement is met if there are constant potential boundary conditions. ${ }^{28}$ However, when the boundary conditions are constant charge, the electric fiel d will not be directed normal to the surface (the surfaces are no longer equipotential) and the stress will not act normal to the surfaces of the tip and substrate. SEI will then underestimate the total force since it does not take the tangential components of the stress into account. Therefore, SEI can only be used to estimate the force between constant charge hemisphere tip and substrate when the radius of the tip is bigger than the Debye length, since the tangential components of the stress will then be small. This method still has advantages over the standard Derjaguin approximation, ${ }^{26}$ in which the force between a hemisphere and plane separated by distance $D$ is approximated by calculating the force per unit area between two infinite planes separated by $D$ and then multiplying by $2 \pi R_{\text {hemisphere. This is only valid when }}$ $R_{\text {hemisphere }}$ is very large and $D$ is very small. The SEI approximation is valid for any value of $D$ as long as

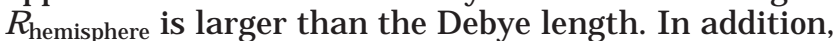
SEI can be used for many geometries and not just hemispheres, while the Derjaguin approximation is only valid for convex tip geometries. The above numerical method was implemented in $C$ and run in Matlab (MathWorks Inc., Natick, MA). Space was discretized to 800 increments (i.e., $\mathrm{N}=800$ ); the program ran until the solution converged sufficiently. ${ }^{34}$

C. Volume Charge Model. Since the CS-GAG molecules are approximately $30 \mathrm{~nm}$ long, they can be modeled as a region of fixed uniform volume charge density using the approach of Ohshima. ${ }^{39}$ Adapted to the MFP geometry, this model represents the tip with SAM layer as a smooth hemi sphere with surface charge, $\sigma_{1}$, and the substrate with CS-GAG as a smooth volume charge density, $\rho_{\mathrm{fix}}$. (Figure 10b) In the electrolyte region outside the fixed volume charge (region I), the PB equation has the form of eq A1.1. In the region inside the fixed volume charge (regi on II), the PB equation has an additional term accounting for the fixed volume density, $\rho_{\text {fix }}$ :

$$
\nabla^{2} \Phi=\frac{2 \mathscr{F} \mathrm{C}_{0}}{\epsilon_{\mathrm{w}}} \sinh \left(\frac{\mathscr{F} \Phi}{\mathrm{RT}}\right)-\frac{\rho_{\mathrm{fix}}}{\epsilon_{\mathrm{w}}}
$$

As a two-region problem, the solution to eq A1.1 in region I and eq A1.8 in region II is subject to boundary conditions at the hemispherical and substrate surfaces and at the edge of the volume charge (Figure 10b). At the surfaces, the boundary conditions are the same as before: the derivative of the potential is proportional to the surface charge density $\left(\partial \Phi / \partial z=\sigma_{1} / \epsilon_{\mathrm{w}}\right.$ at the tip and $\partial \Phi / \partial z=-\sigma_{2} / \epsilon_{\mathrm{w}}=0$ at the substrate, since there is nolonger a surface charge due to the surface monolayer there). There may be some induced surface charge on the substrate but that charge is negligible when compared to the volume charge density due to the CS-GAG and the surface charge density due to the SAM. At the interface between the CS-GAG vol ume charge density and the electrolyte phase, the potential and its derivative (the electric field) must be continuous. When the distance between the surfaces, D, is less than the height 


\section{SURFACE CHARGE MODEL}

(a)

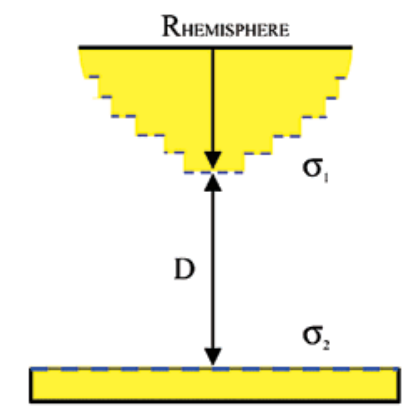

VOLUME CHARGE MODEL

(b)

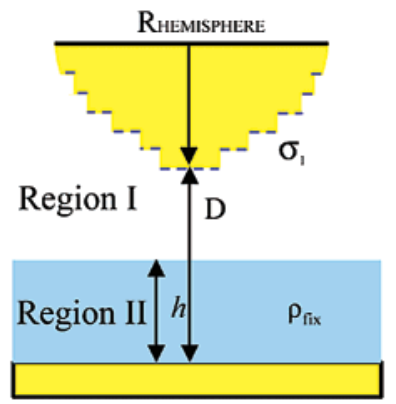

Figure 10. (a) Constant surface charge model (Appendix 1, parts $A$ and $B$ ) and (b) constant volume charge model (Appendix 1C).

of the volume charge, $h$, and since we assume no interdigitation of the molecules, the model reduces to a single region containing a fixed charge density. The PB equation in this case has the form:

$$
\nabla^{2} \Phi=\frac{2 \mathscr{F} \mathrm{C}_{0}}{\epsilon_{\mathrm{w}}} \sinh \left(\frac{\mathscr{F} \Phi}{\mathrm{RT}}\right)-\frac{\rho_{\mathrm{fix}}{ }^{\prime}}{\epsilon_{\mathrm{w}}}
$$

where $\rho_{\mathrm{fix}}{ }^{\prime}=\rho_{\mathrm{fix}}(\mathrm{h} / \mathrm{D})$. While the PB equation is nonlinear, the problem is still one-dimensional due to symmetry, and thus, it can be solved numerically using a similar method as above. This numerical method was also implemented in $\mathrm{C}$ and run in Matlab. Space was discretized to 800 increments (i.e., $\mathrm{N}=800$ ), and the program ran until the solution converged sufficiently.

\section{Nomenclature}

A

$\mathrm{C}_{0}$

$\mathrm{D}$

$\mathscr{F}$

$\mathrm{F}$

F electrostatic

$\mathrm{F}_{\text {flat }}$

$F_{\text {hemisphere }}$

$\mathrm{F}_{\text {hydration }}$

$F_{\text {steric }}$

FvDW

$\mathrm{h}$
Hamaker constant for van der Waals interaction, J

bulk concentration of ions, $\mathrm{mol} / \mathrm{m}^{3}$

intersurface separation distance, $\mathrm{nm}$

Faraday's constant, 96500 C/mol

intersurface force, $\mathrm{nN}$

intersurface electrical double layer force, $\mathrm{nN}$

electrostatic double layer force between two flat surfaces (surface charge model), nN

electrostatic double layer force between charged tip and hemispherical surface (surface charge model), $\mathrm{nN}$

intersurface hydration force, $\mathrm{nN}$

steric force of polymer brush, $\mathrm{nN}$

intersurface van der Waals force, $\mathrm{nN}$

height of volume charge in volume charge model, $\mathrm{nm}$

increment in numerical simulations, unitless
IOLS inverse optical lever sensitivity, $\mathrm{nm} / \mathrm{N}$

$\mathrm{k}_{\mathrm{c}} \quad$ cantilever spring constant, $\mathrm{N} / \mathrm{m}$

$\mathrm{L}_{\text {contour }}$ macromolecular fully extended, contour length, $35 \mathrm{~nm}$

$\mathrm{M}_{\mathrm{n}} \quad$ number average molecular weight, unitless

$\mathrm{N}$ no. of discretizations in numerical simulation, unitless

$\mathrm{R} \quad$ radius of sphere in inverse power law for van der Waals force, $\mathrm{nm}$

$\mathrm{R}_{\text {tip }} \quad$ probe tip radius measured experimentally, $\mathrm{nm}$

$\mathrm{R}_{\text {hemisphere }}$ radius of hemisphere representing probe tip in surface charge model, $\mathrm{nm}$

$\mathrm{S} \quad \mathrm{T}-\mathrm{B} / \mathrm{T}+\mathrm{B}$ output from position sensitive photodiode, $\mathrm{V}$

absolute temperature, $=298 \mathrm{~K}$

z-piezo distance, $\mathrm{nm}$

direction of the force for model calculations, $\mathrm{nm}$

$\mathrm{Z}_{0}$

position coordinate in model calculations, $\mathrm{nm}$

Greek Symbols

$\epsilon_{\mathrm{w}} \quad$ dielectric permittivity of water, $6.9238 \times$ $10^{-10} \mathrm{C} / \mathrm{Nm}^{2}$

$\Phi \quad$ electrostatic potential, $\mathrm{V}=\mathrm{nm} / \mathrm{C}=\mathrm{J} / \mathrm{C}$

$\kappa^{-1} \quad$ electrical interaction Debye length, $\mathrm{nm}$

$\rho_{\text {fix }} \quad$ fixed charge density for volume charge model, $\mathrm{C} / \mathrm{m}^{3}$

$\rho_{\mathrm{fix}}{ }^{\prime} \quad$ fixed charge density for volume charge model when volume is compressed, $\mathrm{C} / \mathrm{m}^{3}$

surface charge/unit area on tip in model, $\mathrm{C} / \mathrm{m}^{2}$ surface charge/unit area on substrate in model, $\mathrm{C} / \mathrm{m}^{2}$

charge regulation parameter, unitless

Abbreviations

AFM atomic force microscopy

DLVO Derjaguin-Landau-Verwey-Overbeek

FDM finite difference method

CS-GAG chondroitin-sulfate glycosaminoglycan

HRFS high-resolution force spectroscopy

IOLS inverse optical lever sensitivity

IS ionic strength

LVDT linear variable differential transformer

MFP molecular force probe

PB Poisson-Boltzmann

PSPD position sensitive photodiode

SAM self-assembled monolayer

SEI Surface element integration

SEM scanning electron microscopy

SFA surface force apparatus

VDW van der Waals

\section{References and Notes}

(1) (a) Buschmann, M. D.; Grodzinsky, A. J . J . Biomech. Eng. 1995, 117, 179-191. (b) J in, M. S.; Grodzinsky, A. J. Macromol ecules 2001, 34, 8330-8339.

(2) Plaas, A. H. K.; West, L. A.; Wong-Palms, S.; Nelson, F. R. T. J . Biol. Chem. 1998, 273, 12642-12649.

(3) Comper, W. D.; Laurent, T. C. Physiol. Rev. 1978, 58, 255315.

(4) de Gennes, P. G. Adv. Colloid Interface Sci. 1987, 27, 189209.

(5) Sukhishvili, S. A.; Granick, S. Langmuir 1997, 13, 49354938. 
(6) Biesalski, M.; Rühe, J . Macromol ecules 1999, 32, 2309-2316.

(7) J ordan, R.; Ulman, A.; Kang, J. F.; Rafailovich, M. H.; Sokolov, J. J . Am. Chem. Soc. 1999, 121, 1016-1022.

(8) Amiel, C.; Sikka, M.; Scheider, J . W.; Tirrell, M. Macromolecules 1995, 28, 3125-3134.

(9) Higashi, N.; Shiba, H.; Niwa, M. Macromolecules 1989, 22, 4650-4652.

(10) Deutsch, A. J .; Midura, R. J .; Plaas, A. H. J . Orthop. Res. 1995, 13, 230-239.

(11) Toida, T.; Shima, M.; Azumaya, S.; Maruyama, T.; Toyoda, H.; I manari, T.; Linhardt, R. J . J . Chromatogr. A. 1997, 787, $266-270$

(12) Hutter, J . L.; Bechhoefer, J . Rev. Sci. Instrum. 1993, 64 1868-1873.

(13) Sarid, D. Scanning Force Microscopy; Oxford University Press: Oxford U.K., 1991.

(14) Yamamoto, S.; Ejaz, M.; Tsujii, Y.; Matsumoto, M.; Fukuda, T. Macromol ecules 2000, 33, 5602-5607.

(15) Yamamoto, S.; Ejaz, M.: Tsujii, Y.; Fukuda, T. Macromolecules 2000, 33, 5608-5612.

(16) Wilbur, J . L.; Kumar, A.; Kim, E.; Whitesides, G. M. Adv. Mater. 1994, 6, 600-604.

(17) Kuettner, K.; Lindenbaum, A. Biochim. Biophys. Acta 1965 $101,223-225$

(18) Freeman, W. D. S. C.; Maroudas, A. Ann. Rheum. Dis. 1975 $34,44-45$.

(19) Sanfeld, A. Thermodynamics of charged and polarized layers: Wiley-interscience publication: Bath, U.K., 1968; Vol. 10.

(20) Kane, V.; Mulvaney, P. Langmuir 1998, 14, 3303-3311.

(21) Vezenov, D. V.; Noy, A.; Rozsnyai, L. F.; Lieber, C. M. J . Am Chem. Soc. 1997, 119, 2006-2015.

(22) Ederth, T.; Claesson, P.; Liedberg, B. Langmuir 1998, 14, $4782-4789$.

(23) Ashby, P. D.; Chen, L.; Lieber, C. M. J . Am. Chem. Soc. 2000, 122, 9467-9472.

(24) Verwey, E. J . W.; Overbeek, J . T. G. Theory of the Stability of Lyophobic Colloids; Elsevier: Amsterdam, 1948.

(25) Derjaguin, B. V.; Landau, L. Acta Physiochim. URSS. 1941, $14,633-662$.

(26) Israelachvili, J. N. Intermolecular and Surface Forces, 2nd ed.; Academic Press: London, 1992.

(27) Derjaguin, B. V. Kolloid Z. 1934, 69, 155-164.

(28) Bhattacharjee, S.; Elimelech, M. J . Colloid Interface Sci. 1997, 193, 273-285.

(29) Parsegian, V. A.; Weiss, G. H. J . Colloid Interface Sci. 1981 81, 285-289.

(30) Biggs, S.; Mulvaney, P.; Zukoski, C. F.; Grieser, F. J . Am. Chem. Soc. 1994, 116, 9150-9157.

(31) Ninham, B. W.; Parsegian, V. A. J . Theor. Biol. 1971, 31 , 405-428.

(32) Parsegian, V. A.; Gingell, D. Biophys. J . 1972, 12, 1192-1204.

(33) Butt, H.-J . Biophys. J . 1991, 60, 777-785

(34) Forsythe, G. E.; Wasow, W. R. Finite Difference Methods for Partial Differential Equations; J ohn Wiley + Sons, Inc.: New York, 1960.

(35) Bain, C. D.; Troughton, E. B.; Tao, Y.-T.; Evall, J .; Whitesides, G. M.; Nuzzo, R. G. J . Am. Chem. Soc. 1989, 111, 321.

(36) (a) Abe, T.; Higashi, N.; Niwa, M.; Kurihara, K. Langmuir 1999, 15, 7725-7731. (b) Hayashi, S.; Abe, T.; Higashi, N.; Niwa, M.; Kurihara, K. Langmuir 2002, 18, 3932-3944.

(37) Maroudas, A. In Adult Articular Cartilage; Freeman, M. A. R., Ed.; Pitman Medical: Kent, U.K., 1979; pp 215-290.

(38) Miller, I.; Williams, D. Phys. Rev. E: Stat. Phys., Plasmas, Fluids, Relat. Interdiscip. Top. 2000, 61, R4706-R4709.

(39) (a) Ohshima, H. Colloid Polym. Sci. 1999, 277, 535-540. (b) Tamashiro, M. N.; Hernández-Zapata, E.; Schorr, P. A. Balastre, M.; Tirrell, M.; Pincus, P. J . Chem. Phys. 2001, 115, 1960-1969.

(40) van Oss, C. I I Interfacial Forces in Aqueous Media; Marcell Dekker: New York, 1994.

(41) Everett, D. H. Basic Principles of Colloid Science; Royal Society of Chemistry: London, 1988.

(42) Sheth, S. R.; Leckband, D. Proc. Natl. Acad. Sci. U.S.A. 1997, 94, 8399-8404.

(43) Kuhl, T. L.; Leckband, D.; Lasic, D. D.; Israelachvili, J . N. Biophys. J . 1994, 66, 1479-1488.
(44) Rijnaarts, H. H. M.; Norde, W.; Lyklema, J .; Zehnder, A. J B. Colloids Surf., B 1999, 14, 179-195.

(45) Fleer, G. J .; Cohen-Stuart, M. A.; Scheutjens, J . M. H. M.; Cosgrove, T.; Vincent, B. Polymers at Interfaces; Chapman and Hall: London, 1993.

(46) Israelachvili, J . N. Adv. Colloid Interface Sci. 1982, 16, 3147.

(47) Pashley, R. M. J . Colloid Interface Sci. 1981, 80, 153-162.

(48) Pashley, R. M. J . Colloid Interface Sci. 1981, 83, 531-546.

(49) Israelachvili, J . N.; Adams, G. E. J . Chem. Soc., Faraday Trans. 1978, 74, 975-1001.

(50) Horn, R. G.; Clarke, D. R.; Clarkson, M. T. J . Mater. Res. 1988, 3, 413-416.

(51) Grabbe, A. Langmuir 1993, 9, 797-801.

(52) Butt, H.-J . Biophys. J . 1991, 60, 1438-1444.

(53) Ducker, W. A.; Senden, T.J .; Pashley, R. M. Nature(London) 1991, 353, 239-241.

(54) Ducker, W. A.; Senden, T. J .; Pashley, R. M. Langmuir 1992 $8,1831-1836$.

(55) Biggs, S.; Chow, M. K.; Zukoski, C. F.; Grieser, F. J . Colloid Interface Sci. 1993, 160, 511-513.

(56) Senden, T. J .; Drummond, C. J . Colloids Surf. A 1995, 94 29-51.

(57) Raiteri, R.; Preuss, M.; Grattarola, M.; Butt, H.-J . Colloids Surf. A 1998, 136, 191-197.

(58) Zhmud, B. V.; Meurk, A.; Bergstrom, L. J . Colloid Interface Sci. 1998, 207, 332-343.

(59) Feldman, K.; Hähner, G.; Spencer, N. D.; Harder, P.; Grunze, M. J . Am. Chem. Soc. 1999, 121, 10134-10141.

(60) Teschke, O.; de Souza, E. F.; Ceotto, G. Langmuir 1999, 15, 4935-4939.

(61) Wang, J .; Bard, A. J . J . Phys. Chem. B 2001, 105, 52175222.

(62) Wang, J .; Bard, A. J . J . Am. Chem. Soc. 2001, 123, 498499.

(63) The Asylum MFP design is such that certain types of highly conducting tips can be grounded via the spring clip that holds the cantilever chip; however, this was not the case for our system. Cleveland, J . Asylum Research; personal communication.

(64) Reiner, E. S.; Radke, C. J . Adv. Colloid Interface Sci. 1993 $47,59-147$

(65) Fixman, M. J . Chem. Phys. 1979, 70, 4995-5005.

(66) Devereux, O. F.; de Bruyn, P. L. Interaction of Plane-Paralle Double Layers; MIT Press: Cambridge, MA, 1963.

(67) Pederson, H. G.; Bergstrom, L.J . Am. Ceram. Soc. 1989, 82, 1137-1145.

(68) Kamiyama, Y.; Israelachvili, J . Macromolecules 1992, 25 , $5081-5088$.

(69) Zauscher, S.; Klingenberg, D. J . J . Colloid I nterface Sci. 2000, 229, 497-510.

(70) Biggs, S.; Burns, J . L.; Yan, Y.; J ameson, G. J .; J enkins, P. Langmuir 2000, 16, 9242-9248.

(71) Le Berre, F.; Malmsten, M.; Blomberg, E. Langmuir 2001 $17,699-704$

(72) Abraham, T.; Giasson, S.; Gohy, J . F.; J erome, R. Langmuir 2000, 16, 4286-4292.

(73) Kelley, T. W.; Schorr, P. A.; J ohnson, K. D.; Tirrell, M.; Frisbie, C. D. Macromolecules 1998, 31, 4297-4300.

(74) Luckham, P. F.; Klein, J. Macromolecules 1985, 18, 721728.

(75) Hale, G.; Hann, A. C.; Wusteman, F. S.; Gacesa, P. Biomaterials 1988, 9, 376-378.

(76) Tran, Y.; Auroy, P.; Lee, L.-T. Macromolecules 1999, 32 8952-8964.

(77) Irvine, D. J .; Mayes, A. M.; Satija, S. K.; Barker, J . G.; SofiaAllgor, S. J .; Griffith, L. G. J . Biomed. Mater. Res. 1998, 40, 498-509.

(78) Fleer, G. J .; Lykema, J. Adsorption from Solution at the Solid-Liquid Interface; Academic Press: New York, 1983.

(79) Buckwalter, J . A.; Rosenberg, L. C. J . Biol. Chem. 1982, 257, 9830-9839.

(80) Hardingham, T. E.; Fosang, A.J . FASEB J . 1992, 6, 861-870. MA0121621 